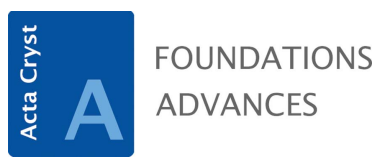

ISSN 2053-2733

Received 7 February 2018

Accepted 30 April 2018

Edited by P. R. Willmott, Swiss Light Source, Switzerland

Keywords: grazing-incidence X-ray diffraction; thin films; indexing; specular scan; mathematical crystallography.

Supporting information: this article has supporting information at journals.iucr.org/a

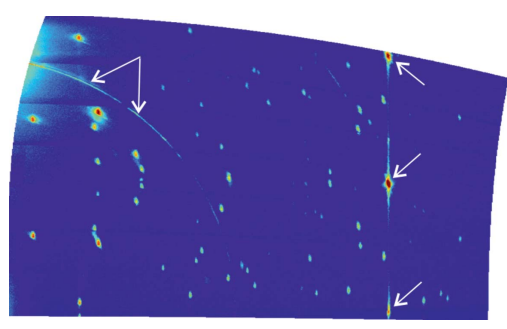

OPEN $\odot$ ACCESS

\section{Indexing of grazing-incidence X-ray diffraction patterns: the case of fibre-textured thin films}

\author{
Josef Simbrunner, ${ }^{\mathrm{a} *}$ Clemens Simbrunner, ${ }^{\mathrm{b}}$ Benedikt Schrode, ${ }^{\mathrm{c}}$ Christian Röthel, \\ Natalia Bedoya-Martinez, ${ }^{\mathrm{C}}$ Ingo Salzmann ${ }^{\mathrm{d}}$ and Roland Resel ${ }^{\mathrm{C}}$
}

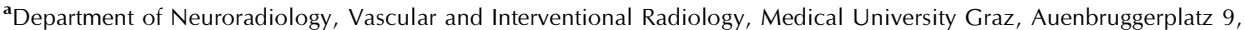
Graz, 8036, Austria, ${ }^{\mathbf{b}} \mathrm{E}+$ E Elektronik Ges.m.b.H., Langwiesen 7, Engerwitzdorf, 4209, Austria, ${ }^{\mathbf{C}}$ Institute of Solid State Physics, Technical University Graz, Petersgasse 16, Graz, 8010, Austria, and d Department of Physics, Department of Chemistry and Biochemistry, Concordia University, 7141 Sherbrooke Street W., SP 265-20, Montreal, Quebec H4B 1R6, Canada. *Correspondence e-mail: josef.simbrunner@medunigraz.at
\end{abstract}

Crystal structure solutions from thin films are often performed by grazingincidence X-ray diffraction (GIXD) experiments. In particular, on isotropic substrates the thin film crystallites grow in a fibre texture showing a well defined crystallographic plane oriented parallel to the substrate surface with random inplane order of the microcrystallites forming the film. In the present work, analytical mathematical expressions are derived for indexing experimental diffraction patterns, a highly challenging task which hitherto mainly relied on trial-and-error approaches. The six lattice constants $a, b, c, \alpha, \beta$ and $\gamma$ of the crystallographic unit cell are thereby determined, as well as the rotation parameters due to the unknown preferred orientation of the crystals with respect to the substrate surface. The mathematical analysis exploits a combination of GIXD data and information acquired by the specular X-ray diffraction. The presence of a sole specular diffraction peak series reveals fibretextured growth with a crystallographic plane parallel to the substrate, which allows establishment of the Miller indices $u, v$ and $w$ as the rotation parameters. Mathematical expressions are derived which reduce the system of unknown parameters from the three- to the two-dimensional space. Thus, in the first part of the indexing routine, the integers $u$ and $v$ as well as the Laue indices $h$ and $k$ of the experimentally observed diffraction peaks are assigned by systematically varying the integer variables, and by calculating the three lattice parameters $a, b$ and $\gamma$. Because of the symmetry of the derived equations, determining the missing parameters then becomes feasible: (i) $w$ of the surface parallel plane, (ii) the Laue indices $l$ of the diffraction peak and (iii) analogously the lattice constants $c, \alpha$ and $\beta$. In a subsequent step, the reduced unit-cell geometry can be identified. Finally, the methodology is demonstrated by application to an example, indexing the diffraction pattern of a thin film of the organic semiconductor pentacenequinone grown on the (0001) surface of highly oriented pyrolytic graphite. The preferred orientation of the crystallites, the lattice constants of the triclinic unit cell and finally, by molecular modelling, the full crystal structure solution of the as-yet-unknown polymorph of pentacenequinone are determined.

\section{Introduction}

The appearance of unknown polymorphs within organic thin films is a well known phenomenon which attracts considerable interest in organic electronics and pharmaceutical science (Jones et al., 2016). Frequently used terms for this type of polymorph include substrate-induced phases, substratemediated phases or thin film phases (Bouchoms et al., 1999; Schiefer et al., 2007; Ehmann \& Werzer, 2014). The presence of an isotropic substrate surface during the crystallization process can induce new types of molecular packing, because 
the substrate acts as a template for the crystallization process. Substrates on which such new polymorphs tend to grow typically exhibit a highly flat surface like oxidized silicon wafers, glass plates or polymer surfaces. There, the deposited organic material crystallizes with a strong preferred orientation showing a well defined crystallographic plane (the socalled contact, or texture plane) parallel to the substrate surface. However, no azimuthal (i.e. in-plane) order between the microcrystallites forming such films is observed due to the isotropic nature of the substrate surfaces. This type of crystalline orientation is called uniplanar texture (Heffelfinger \& Burton, 1960) or fibre texture (Roe \& Krigbaum, 1964).

Crystal structure solutions for such thin films are typically performed by grazing-incidence X-ray diffraction (GIXD); the experimental geometry is schematically shown in Fig. 1(a). The primary X-ray beam with the wavevector $\mathbf{k}_{0}$ and the scattered X-ray beam with the wavevector $\mathbf{k}$ determine the scattering vector $\mathbf{q}$ by $\mathbf{q}=\mathbf{k}-\mathbf{k}_{0}$. According to the Laue equation, diffraction occurs if the scattering vector $\mathbf{q}$ is equal to a reciprocal-lattice vector $\mathbf{g}$. For organic crystallites in fibretextured films, the reciprocal-lattice points lie on concentric circles, as illustrated by red circles in Fig. 1(b). Keeping the sample fixed in space, a GIXD experiment then equals a cut through the three-dimensional reciprocal space, roughly perpendicular to the rings of reciprocal-lattice points, and a corresponding two-dimensional reciprocal-space map is obtained [compare Fig. 1(b)]. Note that for thin films with defined in-plane alignment of the crystallites [e.g. if grown on anisotropic substrates like graphene (Salzmann et al., 2012)] or for samples with weak statistics, the system can be artificially reduced to a fibre texture simply by a $360^{\circ}$ rotation around the substrate normal (Röthel, 2017).
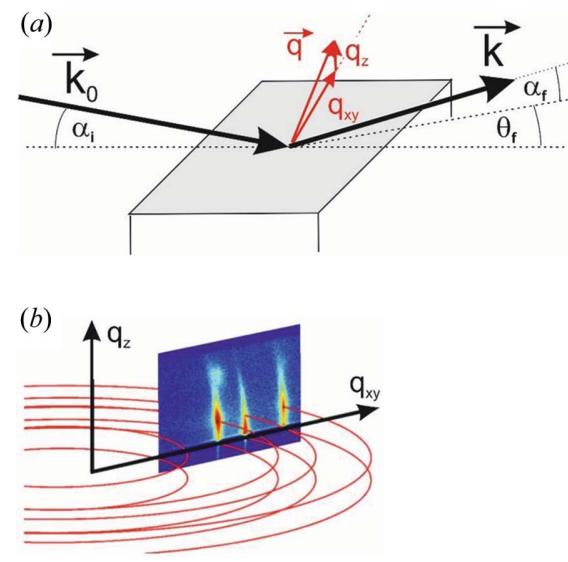

Figure 1

(a) Geometry of a grazing-incidence X-ray diffraction (GIXD) experiment with $\mathbf{k}_{0}$ and $\mathbf{k}$ representing the wavevectors of the primary and of the scattered X-ray beam, respectively, together with the corresponding angle of incidence $\alpha_{\mathrm{i}}$, the in-plane scattering angle $\theta_{\mathrm{f}}$ and the out-of-plane scattering angle $\alpha_{\mathrm{f}}$. The corresponding scattering vector $\mathbf{q}$ is split into an in-plane part $q_{x y}$ and an out-of-plane part $q_{z}$. (b) A reciprocal-space map measured in GIXD geometry plotted as a function of $q_{x y}$ and $q_{z}$ using a colour code for the measured intensity. The reciprocal-lattice points of the thin film crystallites grown in a fibre texture with a fibre axis oriented in the $z$ direction degenerate to concentric rings around that axis.
Crystal structure solutions from GIXD require the indexing of the diffraction pattern, that is, the assignment of Laue indices to the observed Bragg peaks. In our specific case of GIXD on fibre-textured films, two components of the reciprocal-lattice vectors - namely $q_{z}$ and $q_{x y}$ - are available for the indexing process (Smilgies \& Blasini, 2007; Hailey et al., 2014). This is considerably different to the indexing procedure employed for single-crystal diffraction patterns, where all three components of reciprocal-lattice vectors are recorded, as well as for powder diffraction of polycrystalline materials, where only the lengths of the scattering vectors are detected. In the case of single-crystal diffraction, three linearly independent reciprocal-lattice vectors are required to span the reciprocal lattice. Any other experimentally determined reciprocal-lattice vector has then to fit into this specific reciprocal lattice. Since complete three-dimensional vectors are used, even indexing of configurations with multiple lattices can be successfully achieved (Jacobson, 1976; Powell, 1999; Breiby et al., 2008; Gildea et al., 2014; Dejoie et al., 2015; Morawiec, 2017). In the case of powder diffraction, only the lengths of the reciprocal-space vectors are used and the unknown variables are then up to six unit-cell parameters (in the case of a triclinic system) and a set of Laue indices with a triple of three integer values each. This problem cannot be solved algebraically. One possibility, however, is the dichotomy method where the cell constants are varied in increasingly smaller intervals and the $h \mathrm{kl}$ indices are subsequently refined using the least-square method (Boultif \& Louër, 1991, 2004). For simplification of the indexing process, boundary conditions can be imposed.

Few programs have yet been developed for the indexing of two-dimensional reciprocal-space maps (Smilgies \& Blasini, 2007; Breiby et al., 2008; Hailey et al., 2014; Jiang, 2015). Certainly, the situation is relatively trivial if all lattice parameters are known. However, for a successful indexing it is still necessary to determine the contact plane of the investigated crystals in fibre-textured films. For this reason, the rotation matrix of the thin film crystallites relative to the substrate surface has to be considered (Shmueli, 2006). If the lattice parameters are, however, unknown, both the lattice constants and the rotation matrix need to be determined, which represents a significantly more challenging task. Present approaches for the indexing of such systems are mainly based on trial and error, which is clearly unsatisfactory for obvious reasons.

Here, we demonstrate the analytical derivation of mathematical expressions to be employed in the indexing of twodimensional reciprocal-space maps. To this end, we use two components of the reciprocal-space vectors, the in-plane part $q_{x y}$ and the out-of-plane part $q_{z}$. A further input parameter for the indexing arises from specular X-ray diffraction experiments, as in essentially all cases of crystalline organic thin films grown in a fibre texture one defined Bragg peak (or one Bragg peak series) is observed at $q_{\text {spec }}$, originating from the plane normal to the fibre axis of the film. This peak (series) is due to diffraction from the contact plane of the fibre-textured film, which is assigned to a crystallographic plane of Miller indices $u, v$ and $w$ (Salzmann \& Resel, 2004; Smilgies \& Blasini, 2007; Hailey et al., 2014; Jiang, 2015). By combining the peak posi- 
Table 1

Relations between the parameters of the direct lattice $(a, b, c, \alpha, \beta, \gamma)$ and of the reciprocal lattice $\left(a^{*}, b^{*}, c^{*}, \alpha^{*}, \beta^{*}, \gamma^{*}\right)$ and the volume of the crystallographic unit cell $V$.

$$
\begin{array}{ccc}
a^{*}=\frac{2 \pi b c \sin \alpha}{V} & \cos \alpha^{*}=\frac{\cos \beta \cos \gamma-\cos \alpha}{\sin \beta \sin \gamma} & \sin \alpha^{*}=\frac{V}{a b c \sin \beta \sin \gamma} \\
b^{*}=\frac{2 \pi a c \sin \beta}{V} & \cos \beta^{*}=\frac{\cos \alpha \cos \gamma-\cos \beta}{\sin \alpha \sin \gamma} & \sin \beta^{*}=\frac{V}{a b c \sin \alpha \sin \gamma} \\
c^{*}=\frac{2 \pi a b \sin \gamma}{V} & \cos \gamma^{*}=\frac{\cos \alpha \cos \beta-\cos \gamma}{\sin \alpha \sin \beta} & \sin \gamma^{*}=\frac{V}{a b c \sin \alpha \sin \beta} \\
V=a b c\left(1-\cos ^{2} \alpha-\cos ^{2} \beta-\cos ^{2} \gamma+2 \cos \alpha \cos \beta \cos \gamma\right)^{1 / 2}=a b c \sin \alpha^{*} \sin \beta \sin \gamma & \\
\cos \alpha=\frac{\cos \beta^{*} \cos \gamma^{*}-\cos \alpha^{*}}{\sin \beta^{*} \sin \gamma^{*}} & \cos \beta=\frac{\cos \alpha^{*} \cos \gamma^{*}-\cos \beta^{*}}{\sin \alpha^{*} \sin \gamma^{*}} & \cos \gamma=\frac{\cos \alpha^{*} \cos \beta^{*}-\cos \gamma^{*}}{\sin \alpha^{*} \sin \beta^{*}}
\end{array}
$$

tions in the GIXD pattern $\left(q_{x y}, q_{z}\right)$ with the specular peak $\left(q_{\mathrm{spec}}\right)$, the required number of unknown parameters for indexing significantly reduces.

If all three components of the scattering vectors are measured, the orientation of the crystal has to be considered by including the rotation parameters. Though the number of equations is smaller than the number of unknowns, the analytical treatment is much more straightforward since it is purely based on linear equations (see Appendix E).

\section{Methods}

For the following mathematical treatise a laboratory coordinate system with the $x y$ plane being parallel to the substrate surface is assumed.

\subsection{Non-rotated case - contact plane (001)}

In the following analysis, $a, b, c, \alpha, \beta$ and $\gamma$ are the parameters of the (direct) unit cell, and $a^{*}, b^{*}, c^{*}, \alpha^{*}, \beta^{*}$ and $\gamma^{*}$ are the reciprocal cell parameters (Giacovazzo, 2011), which are summarized in Table 1.

If the (001) lattice plane is parallel to the substrate surface in a GIXD experiment, the reciprocal-lattice vector $\mathbf{g}$ with its Laue indices $h, k$ and $l$ can be represented by the equation

$$
\mathbf{g}=\left(\begin{array}{l}
g_{x} \\
g_{y} \\
g_{z}
\end{array}\right)=\mathbf{A}_{001}^{*}\left(\begin{array}{l}
h \\
k \\
l
\end{array}\right)
$$

where the matrix $\mathbf{A}_{001}^{*}$ is given as

$$
\mathbf{A}_{001}^{*}=\left(\begin{array}{ccc}
a^{*} \sin \beta^{*} \sin \gamma & 0 & 0 \\
-a^{*} \sin \beta^{*} \cos \gamma & b^{*} \sin \alpha^{*} & 0 \\
a^{*} \cos \beta^{*} & b^{*} \cos \alpha^{*} & c^{*}
\end{array}\right) .
$$

When the Laue condition $\mathbf{q}=\mathbf{g}$ is fulfilled, diffraction can be observed.

In the real space, $\mathbf{A}_{001}$ characterizes the matrix of the lattice vectors $\mathbf{a}_{0}, \mathbf{b}_{0}$ and $\mathbf{c}_{0}$, which is in the non-rotated system given by

$$
\mathbf{A}_{001}=\left(\begin{array}{c}
\mathbf{a}_{0} \\
\mathbf{b}_{0} \\
\mathbf{c}_{0}
\end{array}\right)=\left(\begin{array}{ccc}
a & 0 & 0 \\
b \cos \gamma & b \sin \gamma & 0 \\
c \cos \beta & -c \sin \beta \cos \alpha^{*} & c \sin \beta \sin \alpha^{*}
\end{array}\right) .
$$

Equations (2) and (3) are connected via

$$
\mathbf{A}_{001}=2 \pi \mathbf{A}_{001}^{*}{ }^{-1} .
$$

The volume $V$ of the unit cell can be calculated by

$$
V=\operatorname{det}\left(\mathbf{A}_{001}\right)=a b c \sin \alpha^{*} \sin \beta \sin \gamma .
$$

Using equation (1) and the relations given in Table 1, the inand out-of-plane components of the reciprocal vector $\mathbf{g}$ can be explicitly written as

$$
\begin{gathered}
g_{x y}^{2}=g_{x}^{2}+g_{y}^{2}=h^{2} z_{a}^{2}+k^{2} z_{b}^{2}-2 h k z_{a} z_{b} \cos \gamma, \\
g_{z}=h a^{*} \cos \beta^{*}+k b^{*} \cos \alpha^{*}+l c^{*},
\end{gathered}
$$

with $z_{a}=2 \pi /(a \sin \gamma)$ and $z_{b}=2 \pi /(b \sin \gamma)$. From equation (6) the unit-cell parameters which are oriented in-plane, namely $a, b$ and $\gamma$, can be determined; in further consequence equation (7) leads to parameters $c, \alpha$ and $\beta$. The integer variables of the Laue indices have to be varied and the values of $q_{x y}$ and $q_{z}$ from three independent Bragg peak series are required to obtain a solution for the corresponding unit-cell parameters, which have to be checked if proper Laue indices can be obtained for all measured diffraction peaks (Truger $e t$ al., 2016). 

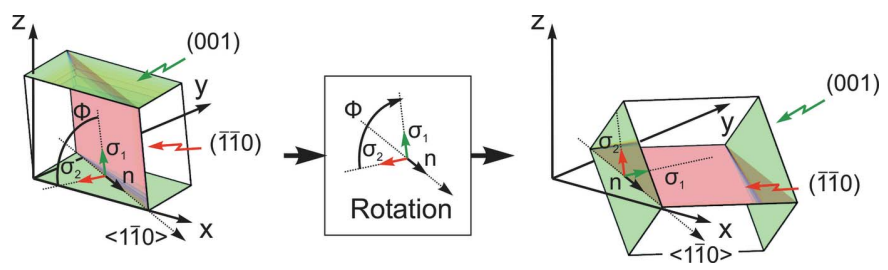

Figure 2

Sketch of a triclinic crystal cell oriented with its (001) net plane parallel to the $x y$ plane. For studying more general orientations with, e.g., a (-1-10) contact plane, all planes and vectors have to be rotated around the zone axis $[1-10]$ by the angle $\Phi$. The zone axis is defined by the vector $\mathbf{n}$, being orthogonal to $\sigma_{1}$ and $\sigma_{2}$. As visualized in the right part of the figure, lattice planes as well as crystallographic directions follow this transformation.

\subsection{Rotated case - contact plane (uvw)}

Obviously, the situation becomes more complex if the (001) lattice plane is not parallel to the substrate surface as the matrix $\mathbf{A}_{001}^{*}$ has now to be transformed. In particular, it has to be rotated around the zone axis which is defined by the (001) plane (characterized by its normal vector $\boldsymbol{\sigma}_{1}$ ) and the new contact plane $(u v w)$, as characterized by its normal vector $\boldsymbol{\sigma}_{2}$. A graphical sketch of the discussed geometry is presented in Fig. 2.

It can easily be proven that an arbitrary rotation of the lattice vectors in the real space corresponds to an identical rotation of the reciprocal-space vectors. If $\mathbf{R}$ is an arbitrarily chosen rotation matrix acting on the lattice vectors and $\mathbf{R}^{-1}=$ $\mathbf{R}^{\mathrm{T}}$ is its inverse, the following relation can be deduced from equation (4):

$$
\begin{aligned}
\mathbf{R}\left(\begin{array}{c}
\mathbf{a}_{0} \\
\mathbf{b}_{0} \\
\mathbf{c}_{0}
\end{array}\right)^{\mathrm{T}} & =\mathbf{R} \mathbf{A}_{001}^{\mathrm{T}}=\left[\mathbf{A}_{001} \mathbf{R}^{\mathrm{T}}\right]^{\mathrm{T}}=2 \pi\left[\mathbf{A}_{001}^{*}{ }^{-1} \mathbf{R}^{\mathrm{T}}\right]^{\mathrm{T}} \\
& =2 \pi\left[\left(\mathbf{R} \mathbf{A}_{001}^{*}\right)^{-1}\right]^{\mathrm{T}} .
\end{aligned}
$$

Therefore, equation (4) can be generalized and written in the form

$$
\mathbf{A}=\left(\begin{array}{l}
\mathbf{a} \\
\mathbf{b} \\
\mathbf{c}
\end{array}\right)=2 \pi \mathbf{A}^{*-1}
$$

where $\mathbf{a}=\mathbf{R} \mathbf{a}_{0}, \mathbf{b}=\mathbf{R} \mathbf{b}_{0}$ and $\mathbf{c}=\mathbf{R} \mathbf{c}_{0}$ are the rotated lattice vectors and $\mathbf{A}^{*}=\mathbf{R} \mathbf{A}_{001}^{*}$.

Based on the graphical representation it can be shown that the unit vector $\mathbf{n}$ of the zone axis is calculated by the vector product of $\sigma_{1}$ and $\sigma_{2}$ :

$$
\begin{aligned}
& \boldsymbol{\sigma}_{1}=\mathbf{A}_{001}^{*}\left(\begin{array}{l}
0 \\
0 \\
1
\end{array}\right), \\
& \boldsymbol{\sigma}_{2}=\mathbf{A}_{001}^{*}\left(\begin{array}{c}
u \\
v \\
w
\end{array}\right),
\end{aligned}
$$

$$
\mathbf{n}=\left(\begin{array}{l}
n_{1} \\
n_{2} \\
n_{3}
\end{array}\right)=\frac{\boldsymbol{\sigma}_{1} \times \boldsymbol{\sigma}_{2}}{\left|\boldsymbol{\sigma}_{1} \times \boldsymbol{\sigma}_{2}\right|} .
$$

The angle of rotation $\Phi$ is obtained by the scalar product

$$
\cos \Phi=\frac{\boldsymbol{\sigma}_{1} \cdot \boldsymbol{\sigma}_{2}}{\left|\boldsymbol{\sigma}_{1}\right|\left|\boldsymbol{\sigma}_{2}\right|}
$$

The matrix $\mathbf{R}$, which describes a rotation by $\Phi$ around the axis $\mathbf{n}$, is given by (Shmueli, 2006)

$$
\begin{aligned}
& \mathbf{R}= \\
& \left(\begin{array}{ccc}
n_{1}^{2}(1-\cos \Phi)+\cos \Phi & n_{1} n_{2}(1-\cos \Phi)+n_{3} \sin \Phi & n_{1} n_{3}(1-\cos \Phi)-n_{2} \sin \Phi \\
n_{1} n_{2}(1-\cos \Phi)-n_{3} \sin \Phi & n_{2}^{2}(1-\cos \Phi)+\cos \Phi & n_{2} n_{3}(1-\cos \Phi)+n_{1} \sin \Phi \\
n_{1} n_{3}(1-\cos \Phi)+n_{2} \sin \Phi & n_{2} n_{3}(1-\cos \Phi)-n_{1} \sin \Phi & n_{3}^{2}(1-\cos \Phi)+\cos \Phi
\end{array}\right) .
\end{aligned}
$$

Combining equations (10) to (12) yields the components of the zone axis unit vector $\mathbf{n}$ :

$$
\begin{aligned}
& n_{1}=\frac{u z_{a} \cos \gamma-v z_{b}}{\left(u^{2} z_{a}^{2}+v^{2} z_{b}^{2}-2 u v z_{a} z_{b} \cos \gamma\right)^{1 / 2}}, \\
& n_{2}=\frac{u z_{a} \sin \gamma}{\left(u^{2} z_{a}^{2}+v^{2} z_{b}^{2}-2 u v z_{a} z_{b} \cos \gamma\right)^{1 / 2}},
\end{aligned}
$$

$n_{3}=0$, which results in the condition $n_{1}^{2}+n_{2}^{2}=1$. In a next step the angle of rotation $\Phi$ can be obtained by combining equations (10), (11) and (13) as

$$
\begin{aligned}
\cos \Phi= & \left(u a^{*} \cos \beta^{*}+v b^{*} \cos \alpha^{*}+w c^{*}\right) \\
& /\left[u^{2} z_{a}^{2}+v^{2} z_{b}^{2}-2 u v z_{a} z_{b} \cos \gamma\right. \\
& \left.+\left(u a^{*} \cos \beta^{*}+v b^{*} \cos \alpha^{*}+w c^{*}\right)^{2}\right]^{1 / 2} .
\end{aligned}
$$

Finally, the reciprocal-lattice vector $\mathbf{g}$ can be written as

$$
\mathbf{g}=\mathbf{R} \mathbf{A}_{001}^{*}\left(\begin{array}{l}
h \\
k \\
l
\end{array}\right)=\mathbf{A}_{\mathbf{u v w}}^{*}\left(\begin{array}{l}
h \\
k \\
l
\end{array}\right) .
$$

From equation (18), the following expressions for the radius $g_{x y z}=\left(g_{x}^{2}+g_{y}^{2}+g_{z}^{2}\right)^{1 / 2}$ and the out-of-plane part $g_{z}$ of the reciprocal-lattice vector can be derived:

$$
\begin{aligned}
g_{x y z}^{2}= & h^{2} z_{a}^{2}+k^{2} z_{b}^{2}-2 h k z_{a} z_{b} \cos \gamma \\
& +\left(h a^{*} \cos \beta^{*}+k b^{*} \cos \alpha^{*}+l c^{*}\right)^{2}, \\
g_{z}= & {\left[h u z_{a}^{2}+k v z_{b}^{2}-(h v+k u) z_{a} z_{b} \cos \gamma\right.} \\
& +\left(u a^{*} \cos \beta^{*}+v b^{*} \cos \alpha^{*}+w c^{*}\right) \\
& \left.\times\left(h a^{*} \cos \beta^{*}+k b^{*} \cos \alpha^{*}+l c^{*}\right)\right] \\
/ & {\left[u^{2} z_{a}^{2}+v^{2} z_{b}^{2}-2 u v z_{a} z_{b} \cos \gamma\right.} \\
& \left.+\left(u a^{*} \cos \beta^{*}+v b^{*} \cos \alpha^{*}+w c^{*}\right)^{2}\right]^{1 / 2} .
\end{aligned}
$$

If the condition $h=u, k=v$ and $l=w$ is fulfilled, equations (19) and (20) are identical, which means that there is only a contribution from the out-of-plane part $g_{z}$, whereas the inplane part $g_{x y}$ is zero. This is valid for the specular scan $g_{\text {spec }}$, which is exactly sensitive to the lattice plane parallel to the surface, and therefore can be explicitly written as 
Table 2

Relations for the total length $g_{x y z}$, the out-of-plane part $g_{z}$ and the in-plane part $g_{x y}$ of the reciprocal-space vectors with indices $h k l$ and of the vector $u v w$ $\left(g_{\text {spec }}\right)$ by using direct- and reciprocal-lattice parameters and the volume $V$.

$$
\begin{aligned}
& g_{x y z}^{2}=g_{x}^{2}+g_{y}^{2}+g_{z}^{2}=h^{2} a^{* 2}+k^{2} b^{* 2}+l^{2} c^{* 2}+2 h k a^{*} b^{*} \cos \gamma^{*}+2 h l a^{*} c^{*} \cos \beta^{*}+2 k l b^{*} c^{*} \cos \alpha^{*} \\
& g_{z} g_{\text {spec }}=h u a^{* 2}+k v b^{* 2}+l w c^{* 2}+(h v+k u) a^{*} b^{*} \cos \gamma^{*}+(h w+l u) a^{*} c^{*} \cos \beta^{*}+(k w+l v) b^{*} c^{*} \cos \alpha^{*} \\
& g_{\text {spec }}=\left(u^{2} a^{* 2}+v^{2} b^{* 2}+w^{2} c^{* 2}+2 u v a^{*} b^{*} \cos \gamma^{*}+2 u w a^{*} c^{*} \cos \beta^{*}+2 v w b^{*} c^{*} \cos \alpha^{*}\right)^{1 / 2} \\
& g_{x y}^{2} g_{\text {spec }}^{2} V^{2} \frac{1}{(2 \pi)^{4}}=(k w-l v)^{2} a^{2}+(h w-l u)^{2} b^{2}+(h v-k u)^{2} c^{2}+2(k u-h v)(h w-l u) b c \cos \alpha \\
& +2(h v-k u)(k w-l v) a c \cos \beta+2(h w-l u)(l v-k w) a b \cos \gamma \\
& g_{x y}^{2}=\left(\frac{2 \pi}{a \sin \gamma}\right)^{2}\left[\left(h-u \frac{g_{z}}{g_{\text {spec }}}\right)^{2}+\left(u \frac{g_{x y}}{g_{\text {spec }}}\right)^{2}\right]+\left(\frac{2 \pi}{b \sin \gamma}\right)^{2}\left[\left(k-v \frac{g_{z}}{g_{\text {spec }}}\right)^{2}+\left(v \frac{g_{x y}}{g_{\text {spec }}}\right)^{2}\right] \\
& -2 \frac{2 \pi}{a \sin \gamma} \frac{2 \pi}{b \sin \gamma} \cos \gamma\left[\left(h-u \frac{g_{z}}{g_{\text {spec }}}\right)\left(k-v \frac{g_{z}}{g_{\text {spec }}}\right)+u v\left(\frac{g_{x y}}{g_{\text {spec }}}\right)^{2}\right]-\frac{(h v-k u)^{2}}{g_{\text {spec }}^{2}}\left(\frac{2 \pi}{a \sin \gamma}\right)^{2}\left(\frac{2 \pi}{b \sin \gamma}\right)^{2} \sin ^{2} \gamma \\
& g_{x y}^{2}=\left(\frac{2 \pi}{a \sin \beta}\right)^{2}\left[\left(h-u \frac{g_{z}}{g_{\text {spec }}}\right)^{2}+\left(u \frac{g_{x y}}{g_{\text {spec }}}\right)^{2}\right]+\left(\frac{2 \pi}{c \sin \beta}\right)^{2}\left[\left(l-w \frac{g_{z}}{g_{\text {spec }}}\right)^{2}+\left(w \frac{g_{x y}}{g_{\text {spec }}}\right)^{2}\right] \\
& -2 \frac{2 \pi}{a \sin \beta} \frac{2 \pi}{c \sin \beta} \cos \beta\left[\left(h-u \frac{g_{z}}{g_{\text {spec }}}\right)\left(l-w \frac{g_{z}}{g_{\text {spec }}}\right)+u w\left(\frac{g_{x y}}{g_{\text {spec }}}\right)^{2}\right]-\frac{(h w-l u)^{2}}{g_{\text {spec }}^{2}}\left(\frac{2 \pi}{a \sin \beta}\right)^{2}\left(\frac{2 \pi}{c \sin \beta}\right)^{2} \sin ^{2} \beta \\
& g_{x y}^{2}=\left(\frac{2 \pi}{b \sin \alpha}\right)^{2}\left[\left(k-v \frac{g_{z}}{g_{\text {spec }}}\right)^{2}+\left(v \frac{g_{x y}}{g_{\text {spec }}}\right)^{2}\right]+\left(\frac{2 \pi}{c \sin \alpha}\right)^{2}\left[\left(l-w \frac{g_{z}}{g_{\text {spec }}}\right)^{2}+\left(w \frac{g_{x y}}{g_{\text {spec }}}\right)^{2}\right] \\
& -2 \frac{2 \pi}{b \sin \alpha} \frac{2 \pi}{c \sin \alpha} \cos \alpha\left[\left(k-v \frac{g_{z}}{g_{\text {spec }}}\right)\left(l-w \frac{g_{z}}{g_{\text {spec }}}\right)+v w\left(\frac{g_{x y}}{g_{\text {spec }}}\right)^{2}\right]-\frac{(k w-l v)^{2}}{g_{\text {spec }}^{2}}\left(\frac{2 \pi}{b \sin \alpha}\right)^{2}\left(\frac{2 \pi}{c \sin \alpha}\right)^{2} \sin ^{2} \alpha
\end{aligned}
$$

$$
\begin{aligned}
g_{\text {spec }}= & {\left[u^{2} z_{a}^{2}+v^{2} z_{b}^{2}-2 u v z_{a} z_{b} \cos \gamma\right.} \\
& \left.+\left(u a^{*} \cos \beta^{*}+v b^{*} \cos \alpha^{*}+w c^{*}\right)^{2}\right]^{1 / 2} .
\end{aligned}
$$

From equations (19) to (21) and by including equation (5), the following expression for the in-plane part $g_{x y}$ can be derived:

$$
\begin{aligned}
& g_{x y}^{2} g_{\text {spec }}^{2} V^{2} \frac{1}{(2 \pi)^{4}} \\
& =(k w-l v)^{2} a^{2}+(h w-l u)^{2} b^{2} \\
& \quad+(h v-k u)^{2} c^{2}+2(k u-h v)(h w-l u) b c \cos \alpha \\
& \quad+2(h v-k u)(k w-l v) a c \cos \beta \\
& \quad+2(h w-l u)(l v-k w) a b \cos \gamma .
\end{aligned}
$$

Furthermore, using equations (20) and (21), equation (19) can be rewritten as

$$
\begin{aligned}
g_{x y z}^{2}= & h^{2} z_{a}^{2}+k^{2} z_{b}^{2}-2 h k z_{a} z_{b} \cos \gamma \\
& +\frac{\left\{g_{z} g_{\text {spec }}-\left[h u z_{a}^{2}+k v z_{b}^{2}-(h v+k u) z_{a} z_{b} \cos \gamma\right]\right\}^{2}}{g_{\text {spec }}^{2}-\left(u^{2} z_{a}^{2}+v^{2} z_{b}^{2}-2 u v z_{a} z_{b} \cos \gamma\right)}
\end{aligned}
$$

and by algebraic transformations the following expression can be derived:

$$
\begin{aligned}
g_{x y}^{2} g_{\text {spec }}^{2}= & z_{a}^{2}\left[u^{2} g_{x y}^{2}+\left(h g_{\text {spec }}-u g_{z}\right)^{2}\right]+z_{b}^{2}\left[v^{2} g_{x y}^{2}+\left(k g_{\text {spec }}-v g_{z}\right)^{2}\right] \\
& -2 z_{a} z_{b} \cos \gamma\left[u v g_{x y}^{2}+\left(h g_{\text {spec }}-u g_{z}\right)\left(k g_{\text {spec }}-v g_{z}\right)\right] \\
& -(h v-k u)^{2} z_{a}^{2} z_{b}^{2} \sin ^{2} \gamma .
\end{aligned}
$$

Equation (24) can be regarded as a generalization of equation (6), additionally including the two rotational integer parameters $u$ and $v$, the specular scan $g_{\text {spec }}$ and the out-of-plane 
part $g_{z}$. For $u=v=0$ it reduces to equation (6) in the nonrotated case.

Equation (24) comprises - in addition to the rotation parameters $u$ and $v$-only the lattice parameters $a, b, \gamma$ and the Laue indices $h$ and $k$. This facilitates the mathematical analysis, where the integer variables can be varied and only three real unknowns have to be calculated. Therefore, we note that when indexing GIXD patterns, the acquisition of a specular scan is of considerable help.

In rare cases, net planes oriented parallel to the substrate surface are characterized by a weak structure factor which inhibits the acquisition of a specular scan (Djuric et al., 2012). In such cases $u$ and $v$ must be assumed to be real (instead of integer) numbers which makes the mathematical analysis more exhaustive and an alternative notation of the rotation matrix may be chosen (see Appendix $A$ ).

In Table 2 we provide a summary of the derived equations and further analogous expressions due to symmetry relationships.

As the Laue indices are integers, they can be systematically varied, whereas the real unknown parameters $a, b$ and $\gamma$ can be calculated from the $q_{x y}$ and $q_{z}$ values of three independent Bragg peak series. This can be achieved analytically by employing proper mathematical substitutions to obtain linear equations (see Appendix $B$ ).

For calculating the remaining cell parameters from equations (20) and (21) the following expression can be derived:

$$
\begin{aligned}
& h a^{*} \cos \beta^{*}+k b^{*} \cos \alpha^{*}+l c^{*} \\
& =\frac{g_{z} g_{\text {spec }}-h u z_{a}^{2}-k v z_{b}^{2}+(h v+k u) z_{a} z_{b} \cos \gamma}{\left(g_{\text {spec }}^{2}-u^{2} z_{a}^{2}-v^{2} z_{b}^{2}+2 u v z_{a} z_{b} \cos \gamma\right)^{1 / 2}} .
\end{aligned}
$$

Equation (25) can be regarded as a generalization of equation (7) to which it reduces for $u=v=0$ in the non-rotated case.

Alternatively, by using the symmetry expressions in Table 2 the parameter sets $\{a, c, \beta, u, w\}$ and $\{b, c, \alpha, v, w\}$ can be determined in an analogous manner as $\{a, b, \gamma, u, v\}$.

If one component of the zone axis, which is the intersection of the planes $(u v w)$ and $(h k l)$, is zero, compact expressions for the reciprocal cell parameters $a^{*}, b^{*}$ and $c^{*}$ can be derived (see Appendix $C$ ).

\section{Discussion - determining the reduced cell}

As discussed by Niggli, the reduced cell is defined by the cell that satisfies the conditions derived from the reduction theory of quadratic forms (Niggli, 1928). Such a cell provides a unique description of the lattice and is characterized independently of lattice symmetry. The main conditions for reduction require that the unit cell is based on the three shortest vectors of the lattice; such a unit cell is then called a Buerger cell (Buerger, 1957). However, this cell may not be unique. An unambiguous unit cell is the so-called reduced cell defined by Niggli (Niggli, 1928; Santoro \& Mighell, 1970). The general criteria for the reduced cells are summarized in Table 3; the complete criteria, which include special condi-
Table 3

General criteria for reduced cells.

General criteria for reduced cell type I (positive reduced cell; all of the angles are $\left.<90^{\circ}\right) \dagger$

$$
a^{2} \leq b^{2} \leq c^{2} ; c \cos \alpha \leq \frac{b}{2} ; c \cos \beta \leq \frac{a}{2} ; b \cos \gamma \leq \frac{a}{2}
$$

General criteria for reduced cell type II (negative reduced cell; all of the angles are $\left.\geq 90^{\circ}\right) \dagger$

$$
\begin{gathered}
a^{2} \leq b^{2} \leq c^{2} ; c|\cos \alpha| \leq \frac{b}{2} ; c|\cos \beta| \leq \frac{a}{2} ; b|\cos \gamma| \leq \frac{a}{2} \\
(b c|\cos \alpha|+a c|\cos \beta|+a b|\cos \gamma|) \leq \frac{a^{2}+b^{2}}{2}
\end{gathered}
$$

$\dagger$ Special criteria if equality signs are valid.

tions, are listed in the International Tables of Crystallography (De Wolff, 2016).

If $\mathbf{a}, \mathbf{b}$ and $\mathbf{c}$ are the lattice vectors of the reduced cell, then every linear combination $\mathbf{a}^{\prime}, \mathbf{b}^{\prime}$ and $\mathbf{c}^{\prime}$ of these vectors

$$
\begin{aligned}
\mathbf{a}^{\prime} & =n_{11} \mathbf{a}+n_{12} \mathbf{b}+n_{13} \mathbf{c}, \\
\mathbf{b}^{\prime} & =n_{21} \mathbf{a}+n_{22} \mathbf{b}+n_{23} \mathbf{c}, \\
\mathbf{c}^{\prime} & =n_{31} \mathbf{a}+n_{32} \mathbf{b}+n_{33} \mathbf{c},
\end{aligned}
$$

where $n_{i j}$ are integers and components of the transformation matrix

$$
\mathbf{N}=\left(\begin{array}{lll}
n_{11} & n_{12} & n_{13} \\
n_{21} & n_{22} & n_{23} \\
n_{31} & n_{32} & n_{33}
\end{array}\right)
$$

can be regarded as a superlattice, which obeys the Laue condition (Santoro et al., 1980). Therefore, in general, any solution that is found when indexing a diffraction pattern must be analysed if it satisfies the conditions of the reduced cell.

In the matrix approach to symmetry (Himes \& Mighell, 1987) $\mathbf{N}$ is represented by one of the 64 symmetry matrices to check if the transformation leads to identity $\left(a^{\prime}=a, b^{\prime}=b, c^{\prime}=c, \alpha^{\prime}=\alpha, \beta^{\prime}=\beta, \gamma^{\prime}=\gamma\right)$.

Equations (26) to (28) can be equivalently written as $\mathbf{A}^{\prime}=$ $\left(\mathbf{a}^{\prime}, \mathbf{b}^{\prime}, \mathbf{c}^{\prime}\right)^{\mathrm{T}}=\mathbf{N A}$. Considering equation (9) the following relations are valid:

$$
\begin{gathered}
\mathbf{A}^{\prime}=\mathbf{N} \mathbf{A}=2 \pi \mathbf{N} \mathbf{A}^{*-1}=2 \pi \mathbf{A}^{*-1} \Leftrightarrow \mathbf{A}^{*}=\mathbf{A}^{* \prime} \mathbf{N}, \\
\mathbf{A}^{*}\left(\begin{array}{l}
h \\
k \\
l
\end{array}\right)=\mathbf{A}^{* \prime} \mathbf{N}\left(\begin{array}{l}
h \\
k \\
l
\end{array}\right)=\mathbf{A}^{* \prime}\left(\begin{array}{c}
h^{\prime} \\
k^{\prime} \\
l^{\prime}
\end{array}\right) \Rightarrow\left(\begin{array}{l}
h^{\prime} \\
k^{\prime} \\
l^{\prime}
\end{array}\right)=\mathbf{N}\left(\begin{array}{l}
h \\
k \\
l
\end{array}\right),
\end{gathered}
$$

where $h^{\prime}, k^{\prime}$ and $l^{\prime}$ are the Laue indices in the transformed system. Thus the transformation $\mathbf{N}$ which converts the lattice vectors is the same as that which converts the Laue indices in the reciprocal space. This is summarized in Table 4. 
Table 4

Relations between the cell parameters $a, b, c, \alpha, \beta, \gamma$, the volume $V$, the Laue indices $h, k, l$ and the Miller indices $u, v, w$ of two crystallographic unit cells linearly transformed by the matrix $\mathbf{N}$.

$$
\begin{gathered}
\left(\begin{array}{lll}
a_{x}^{\prime} & a_{y}^{\prime} & a_{z}^{\prime} \\
b_{x}^{\prime} & b_{y}^{\prime} & b_{z}^{\prime} \\
c_{x}^{\prime} & c_{y}^{\prime} & c_{z}^{\prime}
\end{array}\right)=\left(\begin{array}{lll}
n_{11} & n_{12} & n_{13} \\
n_{21} & n_{22} & n_{23} \\
n_{31} & n_{32} & n_{33}
\end{array}\right)\left(\begin{array}{lll}
a_{x} & a_{y} & a_{z} \\
b_{x} & b_{y} & b_{z} \\
c_{x} & c_{y} & c_{z}
\end{array}\right) \\
a^{\prime}=\left|\mathbf{a}^{\prime}\right|=\left(n_{11}^{2} a^{2}+n_{12}^{2} b^{2}+n_{13}^{2} c^{2}+2 n_{11} n_{12} a b \cos \gamma+2 n_{11} n_{13} a c \cos \beta+2 n_{12} n_{13} b c \cos \alpha\right)^{1 / 2} \\
b^{\prime}=\left|\mathbf{b}^{\prime}\right|=\left(n_{21}^{2} a^{2}+n_{22}^{2} b^{2}+n_{23}^{2} c^{2}+2 n_{21} n_{22} a b \cos \gamma+2 n_{21} n_{23} a c \cos \beta+2 n_{22} n_{23} b c \cos \alpha\right)^{1 / 2} \\
c^{\prime}=\left|\mathbf{c}^{\prime}\right|=\left(n_{31}^{2} a^{2}+n_{32}^{2} b^{2}+n_{33}^{2} c^{2}+2 n_{31} n_{32} a b \cos \gamma+2 n_{31} n_{33} a c \cos \beta+2 n_{32} n_{33} b c \cos \alpha\right)^{1 / 2}
\end{gathered}
$$$$
\cos \gamma^{\prime}=\frac{n_{11} n_{21} a^{2}+n_{12} n_{22} b^{2}+n_{13} n_{23} c^{2}+\left(n_{11} n_{22}+n_{12} n_{21}\right) a b \cos \gamma+\left(n_{11} n_{23}+n_{13} n_{21}\right) a c \cos \beta+\left(n_{12} n_{23}+n_{13} n_{22}\right) b c \cos \alpha}{\left|\mathbf{a}^{\prime}\right|\left|\mathbf{b}^{\prime}\right|}
$$$$
\cos \beta^{\prime}=\frac{n_{11} n_{31} a^{2}+n_{12} n_{32} b^{2}+n_{13} n_{33} c^{2}+\left(n_{11} n_{32}+n_{12} n_{31}\right) a b \cos \gamma+\left(n_{11} n_{33}+n_{13} n_{31}\right) a c \cos \beta+\left(n_{12} n_{33}+n_{13} n_{32}\right) b c \cos \alpha}{\left|\mathbf{a}^{\prime}\right|\left|\mathbf{c}^{\prime}\right|}
$$$$
\cos \alpha^{\prime}=\frac{n_{21} n_{31} a^{2}+n_{22} n_{32} b^{2}+n_{23} n_{33} c^{2}+\left(n_{21} n_{32}+n_{22} n_{31}\right) a b \cos \gamma+\left(n_{21} n_{33}+n_{23} n_{31}\right) a c \cos \beta+\left(n_{22} n_{33}+n_{23} n_{32}\right) b c \cos \alpha}{\left|\mathbf{b}^{\prime}\right|\left|\mathbf{c}^{\prime}\right|}
$$

$$
\begin{aligned}
& V^{\prime}=\left\|\begin{array}{lll}
n_{11} & n_{12} & n_{31} \\
n_{21} & n_{22} & n_{23} \\
n_{31} & n_{32} & n_{33}
\end{array}\right\| V \\
& \left(\begin{array}{l}
h^{\prime} \\
k^{\prime} \\
l^{\prime}
\end{array}\right)=\left(\begin{array}{lll}
n_{11} & n_{12} & n_{13} \\
n_{21} & n_{22} & n_{23} \\
n_{31} & n_{32} & n_{33}
\end{array}\right)\left(\begin{array}{l}
h \\
k \\
l
\end{array}\right) \\
& \left(\begin{array}{c}
u^{\prime} \\
v^{\prime} \\
w^{\prime}
\end{array}\right)=\left(\begin{array}{lll}
n_{11} & n_{12} & n_{13} \\
n_{21} & n_{22} & n_{23} \\
n_{31} & n_{32} & n_{33}
\end{array}\right)\left(\begin{array}{c}
u \\
v \\
w
\end{array}\right)
\end{aligned}
$$

Therefore, reduction of the cell parameters to the reduced cell (Santoro \& Mighell, 1970; Mighell, 1976; Křivý \& Gruber, 1976) is equivalent to converting the Laue indices as in the common reciprocal metric tensor approach (Kroll et al., 2011). If there are two solutions to a diffraction pattern with the Laue indices $h, k, l$ of the unitary cell and $h^{\prime}, k^{\prime}, l^{\prime}$ of a superlattice, the transformation matrix $\mathbf{N}$ can be easily obtained by linearly independent Laue indices of three reflections:

$$
\mathbf{N}=\left(\begin{array}{ccc}
h_{1}^{\prime} & h_{2}^{\prime} & h_{3}^{\prime} \\
k_{1}^{\prime} & k_{2}^{\prime} & k_{3}^{\prime} \\
l_{1}^{\prime} & l_{2}^{\prime} & l_{3}^{\prime}
\end{array}\right)\left(\begin{array}{ccc}
h_{1} & h_{2} & h_{3} \\
k_{1} & k_{2} & k_{3} \\
l_{1} & l_{2} & l_{3}
\end{array}\right)^{-1}
$$

The Miller indices $u, v$ and $w$ can equally be used. If the determinant of the transformation matrix equals \pm 1 , the cell volume does not change. Thus, systematically combining three linearly independent triples of Laue indices, respectively, and calculating their determinants can give an estimate of whether a found solution may match the Buerger cell. Furthermore, in GIXD, after finding a set of cell parameters, by calculating three linearly independent reciprocal vectors and evaluating their inverse matrix the three shortest lattice vectors can be determined (see Appendix E).

The criteria for reduced cells demand that $a^{2} \leq b^{2} \leq c^{2}$ and that the angles are either acute (type I) or obtuse (type II). For this, the expressions in Table 5, which directly result from the symmetric properties of the equations in Table 2, are helpful.

\section{Example: pentacenequinone on highly oriented pyrolytic graphite}

We now employ our novel formalism in the indexing of a thin film of 6,13-pentacenequinone ( $\mathrm{PQ}, \mathrm{C}_{22} \mathrm{H}_{12} \mathrm{O}_{2}$ ), which was grown on a freshly cleaved, highly oriented pyrolytic graphite (HOPG) substrate by physical vapour deposition under high vacuum conditions (base pressure $<5 \times 10^{-6} \mathrm{~Pa}$; deposition 
Table 5

(a) Interchangeability of the Miller indices $u, v, w$ and the crystallographic unit-cell parameters $a, b, c, \alpha, \beta, \gamma ;(b)$ change of sign of the Miller indices $u, v, w$ : effects on the crystallographic unit-cell angles $\alpha, \beta, \gamma$ and the Laue indices $h, k, l$.

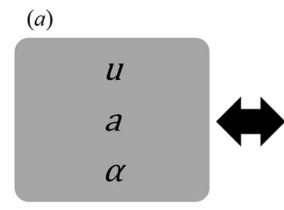

(b)

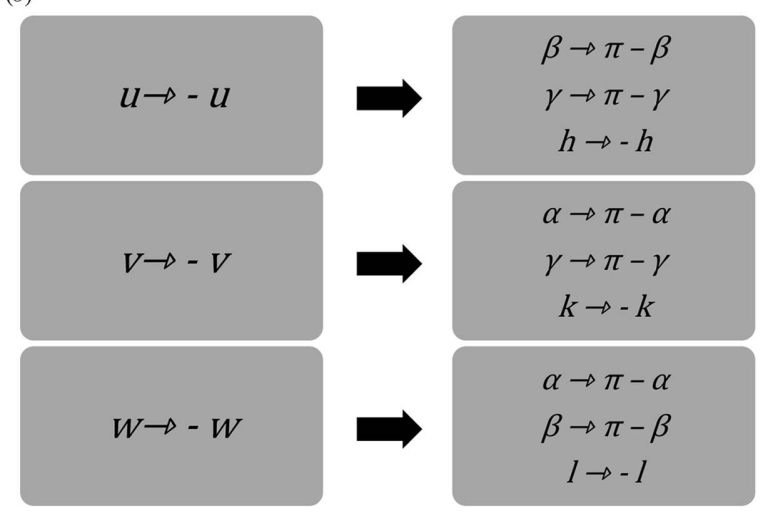

rate $0.5 \mathrm{~nm} \mathrm{~min}^{-1}$; final nominal film thickness $30 \mathrm{~nm}$, as determined by a quartz crystal microbalance). The film was then investigated at the beamline W1 at the synchrotron radiation source DORIS (DESY, HASYLAB, Germany). GIXD experiments together with specular X-ray diffraction were performed using a goniometer in pseudo $2+2$ geometry by a one-dimensional detector (MYTHEN, Dectris) and a wavelength of $1.1796 \AA$ for the primary X-ray beam. The specular scan was performed in the $2 \theta$ range of $2^{\circ}\left(q_{z}=\right.$ $\left.0.185 \AA^{-1}\right)$ to $26^{\circ}\left(2.395 \AA^{-1}\right)$. For the GIXD experiments, the incident angle of the primary beam was set to $\alpha_{\mathrm{i}}=0.13^{\circ}$. The in-plane scattering angle $\theta_{\mathrm{f}}$ was varied between $3^{\circ}$ and $40^{\circ}$ in steps of $0.05^{\circ}$ where for every step an out-of-plane scattering range of $\Delta \alpha_{\mathrm{f}}=3.5^{\circ}$ was recorded. In total, seven scans along $\theta_{\mathrm{f}}$ were performed so that the complete covered angular range of $\alpha_{\mathrm{f}}$ was $0^{\circ}$ to $24.5^{\circ}$. The diffraction pattern was transformed from real to reciprocal space using the custom-made software PyGID (Moser, 2012). The resulting reciprocal-space map illustrates measured intensities on a logarithmic scale by a colour code. The exact positions of the Bragg peaks in terms of $q_{x y}$ and $q_{z}$ were determined by integration of the intensities along $q_{x y}$ and $q_{z}$, respectively, and fitted by Gaussian curves. The $q_{z}$ values of the peak positions were corrected in terms of refraction effects; a maximum variation of $0.011 \AA^{-1}$ was obtained (Resel et al., 2016).

Fig. 3 shows the specular diffraction pattern where only the region around the two dominant diffraction peaks is depicted. The peak at $q_{z}=1.873 \AA^{-1}(d=3.355 \AA)$ agrees well with the expected peak position of the 002 reflection of graphite $(d=$ $3.354 \AA$ ) based on the lattice constants of $a=2.459$ and $c=$ $6.708 \AA$ (Baskin \& Meyer, 1955). The second peak located at $q_{\text {spec }}=1.946 \AA^{-1}(d=3.229 \AA)$ is assigned to the PQ crystals.

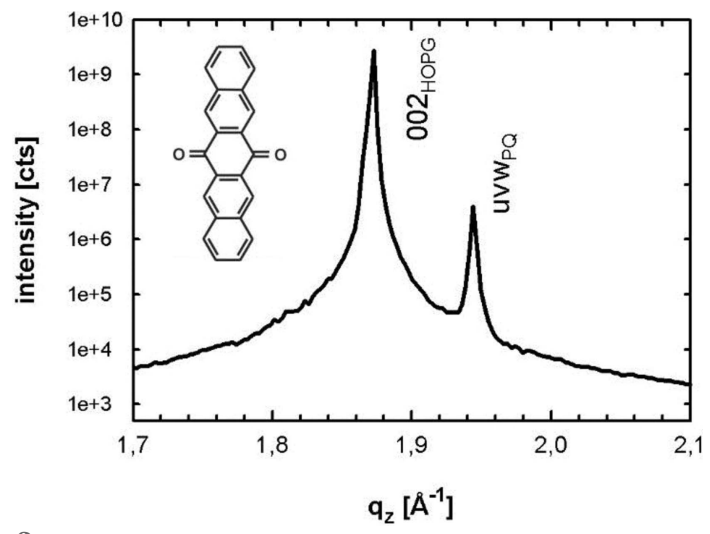

Figure 3

Specular X-ray diffraction of a crystalline thin film of PQ grown on HOPG. The inset gives the chemical structure of the molecule.

Fig. 4(a) shows the diffraction pattern of the GIXD experiment. Bragg peaks at $q_{x y}=2.946 \AA^{-1}$ and $q_{z}=0.002 \AA^{-1}, q_{z}=$ $0.941 \AA^{-1}$ and $q_{z}=1.880 \AA^{-1}$ are identified as the $10-10$, $10-11$ and $10-12$ reflections of the HOPG single-crystal substrate. Additionally, a Debye-Scherrer ring appears at $q=$ $1.87 \AA^{-1}$ which is assigned to disordered 0002 planes of graphite. The diffraction features of the HOPG substrate are marked by arrows in Fig. 4(a).

The remaining Bragg peaks are assigned to PQ crystals; they are distributed within the whole reciprocal-space map. The most intense peaks with their $q_{x y}$ and $q_{z}$ positions were used together with $q_{\text {spec }}=1.946 \AA^{-1}$ for the indexing routine. A total of 74 reflections of the GIXD map were included in the analysis. In a first step of indexing the Miller indices $u$ and $v$ of the contact plane (the crystallographic plane which is parallel to the substrate surface) are varied by integer variables together with a systematic change of the Laue indices of three reflections so that a first set of lattice constants $a, b$ and $\gamma$ are obtained [see equation (43) in Appendix B]. Note that with the restriction of linear independency due to linear transformation [see equation (32)] three pairs of Laue/Miller indices are, in principle, freely eligible to get a mathematically valid solution which may represent a superlattice. This first set of lattice constants is used to determine the Laue indices $h$ and $k$ of all other peak positions $\left(q_{x y}, q_{z}\right)$ until a suitable assignment of the Laue indices $h$ and $k$ to all 74 reflections is obtained. For this procedure, mathematical expressions in Appendix $D$ are helpful. In total 150 integer variables and three real numbers have to be determined.

In a subsequent step, the assignment of the remaining Miller index $w$ as well as the Laue indices $l$ and the evaluation of the lattice constants $c, \alpha$ and $\beta$ have to be accomplished. There are two possible ways. The first possibility relies on symmetry considerations of equation (24) (see Table 2). A systematic exchange of the two Miller indices, the pairs of Laue indices and the three lattice constants leads to a set of three equivalent equations where, finally, all parameters of the indexing are determined. The second possibility is simply using equations (21) and (25), where the remaining integer $w$ of the contact plane, the lattice constants $c, \alpha$ and $\beta$ as well as the Laue indices $l$ of the 74 reflections are obtained. Expressions in 

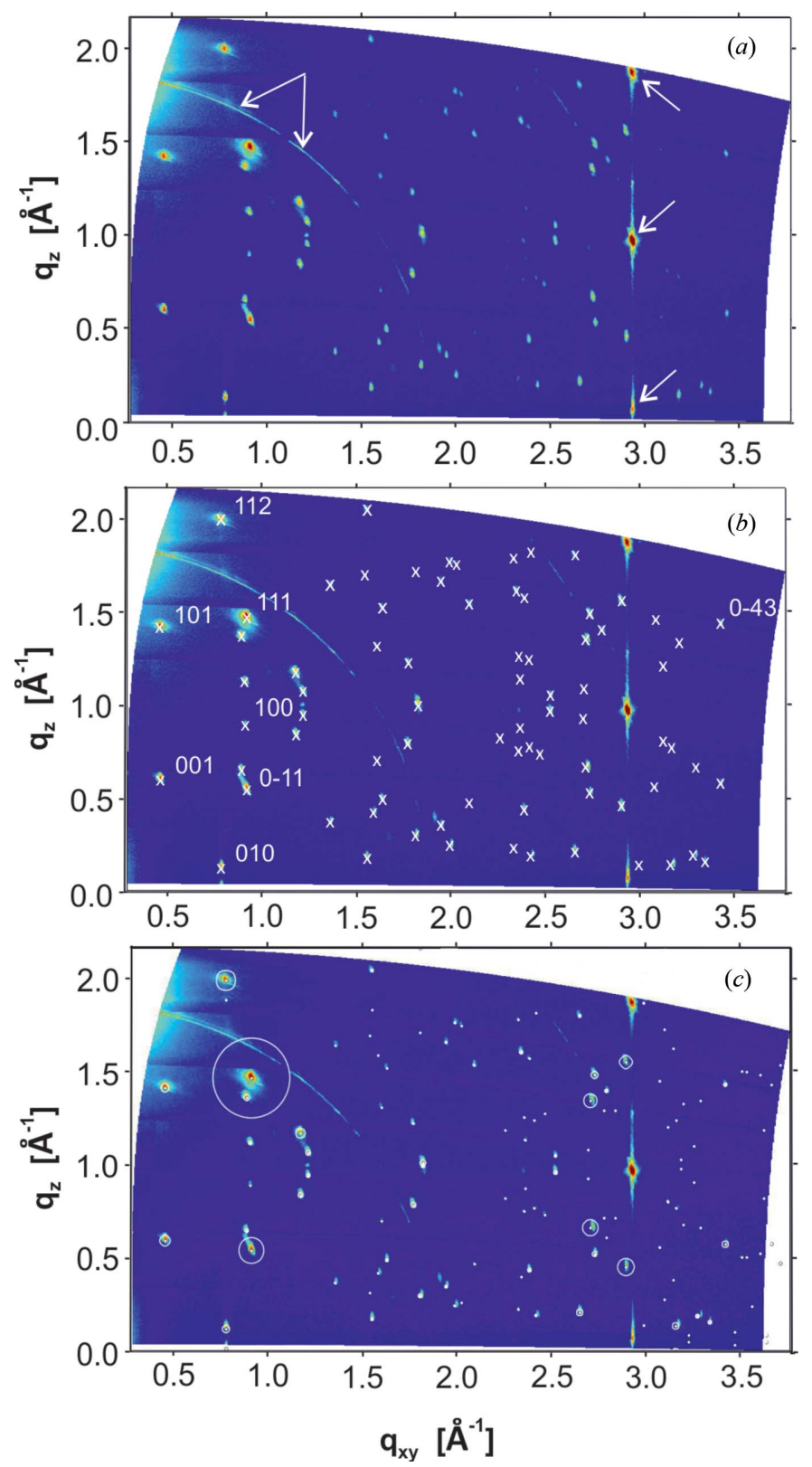

Figure 4

(a) Reciprocal-space map (RSM) of the PQ thin film grown on HOPG (cf. Fig. 3); arrows indicate diffraction features of the substrate. (b) Indexing of the RSM. Crosses denote calculated peak positions assigned to experimentally observed peaks; for clarity, Laue indices are given only for selected Bragg peaks. (c) RSM with calculated peak intensities obtained from the theoretically determined molecular packing; the area of the circles corresponds to the square of the structure factors.

Appendix $C$ can be helpful in determining the parameters $c^{*}$, $w$ and $l$ of specific reflections. In a last step, when all integer variables have been assigned, the values of the real lattice parameters can be fitted. For this procedure expressions in Table 2 can be used.

As the underlying equations do not allow a unique mathematical solution, a manifold of possible results exist. But crystallographic restrictions constrain these mathematical solutions. The cell parameters must obey the scalar product (Niggli) criteria (see Table 3). Furthermore, one has to check if
Table 6

Indexing of the reciprocal-space map of PQ crystals on HOPG (0001) substrate: corresponding Laue indices of selected individual Bragg peaks for solution $1\left(h_{1} k_{1} l_{1}\right)$ with contact plane (102) and for solution $2\left(h_{2} k_{2} l_{2}\right)$ with contact plane $(12-2)$.

\begin{tabular}{lrllll}
\hline$h_{1}$ & $k_{1}$ & $l_{1}$ & $h_{2}$ & $k_{2}$ & $l_{2}$ \\
\hline 0 & 0 & 1 & 0 & 1 & -1 \\
1 & 0 & 1 & 1 & 1 & -1 \\
1 & -1 & 1 & 1 & 0 & -2 \\
0 & 1 & 2 & 0 & 3 & -1 \\
0 & -1 & 2 & 0 & 1 & -3 \\
1 & 0 & 0 & 1 & 0 & 0 \\
1 & 1 & 0 & 1 & 1 & 1 \\
1 & -1 & 0 & 1 & -1 & -1 \\
0 & 1 & 1 & 0 & 2 & 0 \\
0 & -1 & 1 & 0 & 0 & -2 \\
0 & 1 & 0 & 0 & 1 & 1 \\
\hline
\end{tabular}

a solution has the shortest possible edges and thus is a Buerger cell.

For illustration, we depict the following two mathematical solutions, both of which obey the scalar product criteria for type-II cells:

Solution 1: $u_{1}=1, v_{1}=0, w_{1}=2 ; a_{1}=5.067 \AA$ 叫 $b_{1}=8.064 \AA$, $c_{1}=8.882 \AA, \alpha_{1}=91.64^{\circ}, \beta_{1}=93.34^{\circ}, \gamma_{1}=94.01^{\circ}, V_{1}=$ $361.2 \AA^{3}$.

Solution 2: $u_{2}=1, v_{2}=2, w_{2}=-2 ; a_{2}=5.067 \AA, b_{2}=$ $11.824 \AA, c_{2}=12.166 \AA, \alpha_{2}=95.53^{\circ}, \beta_{2}=90.22^{\circ}, \gamma_{2}=95.25^{\circ}, V_{2}$ $=722.4 \AA^{3}$.

In Table 6, corresponding Laue triples of some lower reflections are given. The determinants of three linearly independent triples of indices give mostly \pm 1 for solution 1 and \pm 2 for solution 2 . The transformation matrix $\mathbf{N}$ which leads from solution 2 to solution 1 can be determined according to equation (32):

$$
\mathbf{N}=\left(\begin{array}{ccc}
1 & 0 & 0 \\
1 & \frac{1}{2} & -\frac{1}{2} \\
0 & \frac{1}{2} & \frac{1}{2}
\end{array}\right),
$$

with $\operatorname{det}(\mathbf{N})=\frac{1}{2}$, establishing the relations between the lattice parameters, the Miller indices of the contact plane and the Laue indices of the 74 Bragg peaks (see Table 3).

By applying equation (18), three reciprocal-lattice vectors $\mathbf{g}$, e.g. of the three Laue triples $(1,0,1),(0,1,1)$ and $(0,1,0)$ for solution 1 , and $(1,1,-1),(0,2,0)$ and $(0,1,1)$ for solution 2 , can be calculated. The determinants of the vector matrices should be as small as possible but not equal to zero. By determining their inverse matrices and by multiplying these with vectors $\mathbf{m}$ $=2 \pi\left(m_{1}, m_{2}, m_{3}\right)^{\mathrm{T}}$, where $m_{i}$ are systematically varied integers between -2 and 2 , lattice vectors can be obtained [see Appendix $E$ with emphasis on equation (71)]. In both cases, listing the lengths of these vectors in ascending order yields $5.067,8.064,8.882,9.219,9.819,9.966,10.134,10.479,11.824$ and 12.166 for the ten shortest vectors. The $z$ components of these vectors are all integer multiples of $2 \pi / g_{\text {spec }}$ (in absolute values $1,0,2,1,1,3,2,1,2,2$, respectively, and thus representing the Miller indices - see the equations in Table 7). Therefore, solution 1 matches the reduced cell, whereas 
Table 7

Unit-cell vectors for the parameters $a, b, c, \alpha, \beta, \gamma$, the Laue indices $h, k, l$ and the Miller indices $u, v, w$ and including the specular scan $\left(g_{\text {spec }}\right)$ for the nonrotated $(a)$ and the rotated $(b)$ case.

(a) Non-rotated case $(u=v=0)$ :

$$
\begin{aligned}
& \mathbf{a}=a\left(\begin{array}{c}
\cos \varphi \\
\sin \varphi \\
0
\end{array}\right) \\
& \mathbf{b}=b\left(\begin{array}{c}
\cos (\varphi+\gamma) \\
\sin (\varphi+\gamma) \\
0
\end{array}\right) \\
& \mathbf{c}=c\left(\begin{array}{c}
r_{c} \cos \left(\varphi+\Omega_{c}\right) \\
r_{c} \sin \left(\varphi+\Omega_{c}\right) \\
\frac{w}{c} \frac{2 \pi}{g_{\mathrm{spec}}}
\end{array}\right) \quad r_{c}=\left[1-\left(\frac{w}{c} \frac{2 \pi}{g_{\mathrm{spec}}}\right)^{2}\right]^{1 / 2} \\
& \cos \Omega_{c}=\frac{1}{r_{c}} \cos \beta
\end{aligned}
$$

(b) Rotated case:

$$
\begin{aligned}
& \mathbf{a}=a\left(\begin{array}{c}
r_{a} \cos \left(\varphi+\psi-\Omega_{a}\right) \\
r_{a} \sin \left(\varphi+\psi-\Omega_{a}\right) \\
\frac{u}{a} \frac{2 \pi}{g_{\mathrm{spec}}}
\end{array}\right) \\
& r_{a}=\left[1-\left(\frac{u}{a} \frac{2 \pi}{g_{\text {spec }}}\right)^{2}\right]^{1 / 2} \\
& \mathbf{b}=b\left(\begin{array}{c}
r_{b} \cos \left(\varphi+\psi+\Omega_{b}\right) \\
r_{b} \sin \left(\varphi+\psi+\Omega_{b}\right) \\
\frac{v}{b} \frac{2 \pi}{g_{\text {spec }}}
\end{array}\right) \\
& r_{b}=\left[1-\left(\frac{v}{b} \frac{2 \pi}{g_{\text {spec }}}\right)^{2}\right]^{1 / 2} \\
& \mathbf{c}=c\left(\begin{array}{c}
r_{c} \cos \left(\varphi+\psi+\Omega_{c}\right) \\
r_{c} \sin \left(\varphi+\psi+\Omega_{c}\right) \\
\frac{w}{c} \frac{2 \pi}{g_{\mathrm{spec}}}
\end{array}\right) \\
& r_{c}=\left[1-\left(\frac{w}{c} \frac{2 \pi}{g_{\mathrm{spec}}}\right)^{2}\right]^{1 / 2} \\
& \cos \Omega_{a}=\frac{1}{r_{a}} \frac{\frac{u}{a} \cos \gamma-\frac{v}{b}}{\left[\left(\frac{u}{a}\right)^{2}+\left(\frac{v}{b}\right)^{2}-2 \frac{u v}{a b} \cos \gamma\right]^{1 / 2}} \\
& \cos \Omega_{b}=\frac{1}{r_{b}} \frac{\frac{u}{a}-\frac{v}{b} \cos \gamma}{\left[\left(\frac{u}{a}\right)^{2}+\left(\frac{v}{b}\right)^{2}-2 \frac{u v}{a b} \cos \gamma\right]^{1 / 2}} \\
& \cos \Omega_{c}=\frac{1}{r_{c}} \frac{\frac{u}{a} \cos \alpha-\frac{v}{b} \cos \beta}{\left[\left(\frac{u}{a}\right)^{2}+\left(\frac{v}{b}\right)^{2}-2 \frac{u v}{a b} \cos \gamma\right]^{1 / 2}} \\
& \sin \psi=\frac{\frac{u}{a} \sin \gamma}{\left[\left(\frac{u}{a}\right)^{2}+\left(\frac{v}{b}\right)^{2}-2 \frac{u v}{a b} \cos \gamma\right]^{1 / 2}} \\
& \cos \psi=\frac{\frac{u}{a} \cos \gamma-\frac{v}{b}}{\left[\left(\frac{u}{a}\right)^{2}+\left(\frac{v}{b}\right)^{2}-2 \frac{u v}{a b} \cos \gamma\right]^{1 / 2}}
\end{aligned}
$$

solution 2 represents a superlattice. The thus obtained vectors of both solutions, though they do not coincide, but are equally rotated, span identical parallelepipeds and result in the same cell parameters $a, b, c, \alpha, \beta$ and $\gamma$. Therefore, solution 2 can be reduced very effectively by the described method.

For evaluating the reliability of powder pattern indexing, a factor $F_{N}$ has been introduced (Smith \& Snyder, 1979). For GIXD we suggest the following factors for assessing the accuracy of the obtained result:

$$
\begin{gathered}
d_{N, x y z}=\frac{1}{N} \sum_{i=1}^{N}\left|\frac{q_{x y z, i}-g_{x y z, i}}{q_{x y z, i}}\right| \\
d_{N, z}=\frac{1}{N} \sum_{i=1}^{N}\left|\frac{q_{z, i}-g_{z, i}}{q_{z, i}}\right|
\end{gathered}
$$

where $N$ is the number of reflections, $\left(q_{x y z, i}, q_{z, i}\right)$ are the measured and $\left(g_{x y z, i}, g_{z, i}\right)$ are the calculated peak positions of the $i$ th reflection. In our case $d_{74, x y z}=0.0022$ and $d_{74, z}=0.0032$. However, it should be emphasized that it is additionally necessary to prove that the obtained unit cell corresponds to the reduced cell.

Since the unit-cell dimensions are considerably different to the three reported phases of PQ (Dzyabchenko et al., 1979; Nam et al., 2010; Salzmann et al., 2011), we can conclude that a new polymorph is found. Based on the reduced cell the peak positions are calculated and plotted in Fig. 4(b). A total of 80 positions of Bragg peaks could be assigned to PQ crystals by their Laue indices.

If the specular scan is not known, it is then an additional unknown parameter in equation (24), which has to be solved numerically by using four pairs of input parameters $q_{x y}$ and $q_{z}$. An alternative way would be to exclude the specular diffrac- 
(a)

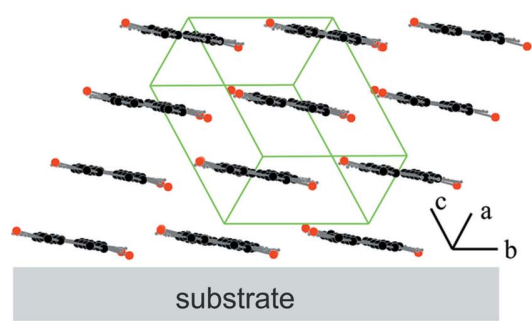

(b)

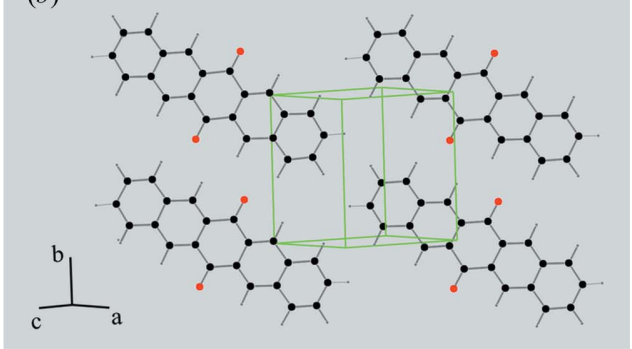

Figure 5

Molecular packing of PQ molecules as well as orientation of the molecules relative to the substrate surface determined from the crystal structure solution: in a side view along the long molecular axis (a) and in a top view of a single molecular layer across the crystallographic (102) plane (b). The crystallographic unit cell is depicted in green.

tion peak from the indexing procedure: an alternative notation of the rotation matrix may then be used [see equation (35) in Appendix $A$ ]. Even in that case a two-step separation of the indexing can be obtained. Input parameters are the total length of the scattering vectors $q_{x y z}$ and $q_{z}$ and the estimated parameters are the lattice constants $a, b, \gamma$ and the two angles $\psi$ and $\varphi$ which express the orientation of the crystal at the substrate surface [equation (38)]. Note that $q_{x y z}$ can be easily determined by $q_{x y z}^{2}=q_{x y}^{2}+q_{z}^{2}$ and due to the Laue condition $q_{x y z}=g_{x y z}$ and $q_{z}=g_{z}$. In our case the rotation angles $\psi=$ $94.01^{\circ}$ and $\varphi=39.78^{\circ}$ are obtained. The other lattice constants $c, \alpha$ and $\beta$ can be obtained from equation (37).

There are different possibilities to determine the molecular packing based on the knowledge of the crystallographic unit cell (David et al., 2006). In the case of organic thin films rigidbody refinement procedures based on experimental structure factors were used (Krauss et al., 2008; Mannsfeld et al., 2011) or theoretical modelling was applied (Schiefer et al., 2007; Jones et al., 2017). Here, the molecular packing relative to the experimentally determined unit cell has been determined by theoretical modelling, where a combination of molecular dynamics (MD) simulations and density functional theory (DFT) was used. MD simulations were carried out using the LAMMPS code in combination with the CHARMM General Force Field v.2b7 (Plimpton, 1995; Vanommeslaeghe et al., 2010). In a first step, several hundred trial structures were created by placing one molecule randomly into a slightly expanded unit cell. During the subsequent MD run, the system was allowed to relax energetically while the unit cell was continuously shrinking to the experimental size. The most promising structures were further redefined using DFT geometry optimizations as implemented in the $V A S P$ package (version 5.4.1) (Kresse \& Hafner, 1993, 1994; Kresse \& Furthmüller, 1996a,b). The Perdew-Burke-Ernzerhof functional for the exchange and correlation (Perdew et al., 1996) and projector-augmented wave potentials for all the elements (Blöchl, 1994; Kresse \& Joubert, 1999) were used. Van der Waals corrections were included following the many-body dispersion approach of Tkatchenko et al. (2012). A planewave cut-off energy of $800 \mathrm{eV}$ and a converged MonkhorstPack grid (Monkhorst \& Pack, 1976) of $7 \times 4 \times 4$ were used. The total energy during the self-consistency loop of each DFT is found; hence, the resulting molecular packing describes the surface-induced phase of PQ on HOPG. The CIF for the solved crystal structure can be found in the supporting information.

The packing of the PQ molecules within the crystal structure can be described by a parallel stacking of the planar molecules. The stacking distance between the planar molecules is about $3.45 \AA$ (Fig. $5 a$ ). Short contacts appear between $\mathrm{O}$ atoms and neighbouring $\mathrm{H}$ atoms and between terminal $\mathrm{H}$ atoms of neighbouring PQ molecules (Fig. 5b). In a subsequent step the orientation of the molecules relative to the substrate surface can be determined. The described indexing routine reveals the assignment of the Laue indices 102 to the specular diffraction. Plotting the crystallographic plane with Miller indices 102 towards the molecular packing of our crystal structure solution directly reveals the orientation of the molecules relative to the substrate surface (Fig. 5a). It is found that the long molecular axes are aligned parallel to the substrate surface. The molecular plane encloses an angle of $8^{\circ}$ to a 'flat-on' orientation. It seems that the enhanced intermolecular interactions via the short oxygen-hydrogen bonds (discussed above) establish a stabilization of a layer formed by tilted molecules.

\section{Conclusion}

In the present work, we provide a unifying framework for the indexing of reciprocal-space maps obtained by GIXD on fibretextured thin films, which we successfully apply in deriving the full structure solution of an as-yet-unknown substrate-mediated polymorph of $\mathrm{PQ}$.

Including the specular peak in the mathematical formalism of diffraction experiments can be of considerable help, especially in the case of GIXD where the spatial orientation of the unit cell has to be considered. For the rotation parameters the integer variables $u, v$ and $w$ can be employed, and mathematical expressions can be derived in which the unknown cell parameters are considerably reduced. This significantly reduces computational efforts, as the integer variables can be systematically varied and only three real unknown parameters remain, which can be analytically calculated using $q_{x y}$ and $q_{z}$ of 
three independent diffraction peaks. In subsequent steps the remaining parameters can then be conveniently determined.

As any linear combination of the unit-cell vectors satisfies the imposed mathematical conditions no unique solution exists. Based on the well known criteria originally imposed by Niggli, the reduced unit cell has therefore to be determined. The main conditions for reduction require that the cell is based on the three shortest vectors of the lattice. These can be obtained from any mathematical solution by the proper threedimensional linear transformation. It may be helpful to use the obtained cell parameters and Laue indices to calculate three linearly independent reciprocal vectors and evaluate their inverse matrix to determine the lattice vectors of the reduced unit cell.

Though our analysis primarily considers the general case of a triclinic system, it also applies to crystal systems of higher symmetries which then imply a higher impact of symmetry considerations such as that of reflection conditions.

\section{APPENDIX $A$}

\section{Alternative notation of the rotation matrix}

If $u$ and $v$ have to be assumed to be real numbers (and if not $u$ $=v=0$ ) it may be more convenient to rewrite equation (14) as

$\mathbf{R}(\psi, \Phi)$
$=\left(\begin{array}{ccc}\cos ^{2} \psi+\cos \Phi \sin ^{2} \psi & \cos \psi \sin \psi(1-\cos \Phi) & -\sin \psi \sin \Phi \\ \cos \psi \sin \psi(1-\cos \Phi) & \sin ^{2} \psi+\cos \Phi \cos ^{2} \psi & \cos \psi \sin \Phi \\ \sin \psi \sin \Phi & -\cos \psi \sin \Phi & \cos \Phi\end{array}\right)$,

where $n_{1}=\cos \psi$ and $n_{2}=\sin \psi$. Then equations (19) and (20) are rewritten as

$$
\begin{aligned}
g_{x y z}^{2}= & h^{2} z_{a}^{2}+k^{2} z_{b}^{2}-2 h k z_{a} z_{b} \cos \gamma \\
& +\left(h a^{*} \cos \beta^{*}+k b^{*} \cos \alpha^{*}+l c^{*}\right)^{2}, \\
g_{z}= & h z_{a} \sin \Phi \cos (\gamma-\psi)-k z_{b} \sin \Phi \cos \psi \\
& +\cos \Phi\left(h a^{*} \cos \beta^{*}+k b^{*} \cos \alpha^{*}+l c^{*}\right),
\end{aligned}
$$

with $z_{a}=2 \pi /(a \sin \gamma)$ and $z_{b}=2 \pi /(b \sin \gamma)$. From equations (36) and (37) the following expression can be derived:

$$
\begin{aligned}
g_{x y z}^{2}= & h^{2} z_{a}^{2}+k^{2} z_{b}^{2}-2 h k z_{a} z_{b} \cos \gamma \\
& +\left[\frac{g_{z}-h z_{a} \sin \Phi \cos (\gamma-\psi)+k z_{b} \sin \Phi \cos \psi}{\cos \Phi}\right]^{2}
\end{aligned}
$$

which comprises the Laue indices $h$ and $k$, the three unit-cell parameters $a, b, \gamma$ and the rotation angles $\Phi$ and $\psi$.

\section{APPENDIX $B$}

Mathematical procedure for analytically determining the cell parameters $a, b$ and $\gamma$

For analytically determining the unit-cell parameters $a, b$ and $\gamma$, it is convenient to introduce the parameters $Z_{a}^{2}, Z_{b}^{2}$ and $X_{\gamma}$ with the substitutional relations

$$
\begin{gathered}
Z_{a}^{2}=z_{a}^{2}\left(1-v^{2} z_{b}^{2} \frac{\sin ^{2} \gamma}{g_{\text {spec }}^{2}}\right), \\
Z_{b}^{2}=z_{b}^{2}\left(1-u^{2} z_{a}^{2} \frac{\sin ^{2} \gamma}{g_{\text {spec }}^{2}}\right), \\
X_{\gamma}=z_{a} z_{b}\left(\cos \gamma-u v z_{a} z_{b} \frac{\sin ^{2} \gamma}{g_{\text {spec }}^{2}}\right) .
\end{gathered}
$$

Note that $Z_{a}^{2}$ and $Z_{b}^{2}$ are always positive. Using these substitutions, equation (24) can be rewritten as

$$
\begin{aligned}
1= & Z_{a}^{2}\left[\frac{1}{g_{x y}^{2}}\left(h-u \frac{g_{z}}{g_{\text {spec }}}\right)^{2}+\frac{u^{2}}{g_{\text {spec }}^{2}}\right] \\
& +Z_{b}^{2}\left[\frac{1}{g_{x y}^{2}}\left(k-v \frac{g_{z}}{g_{\text {spec }}}\right)^{2}+\frac{v^{2}}{g_{\text {spec }}^{2}}\right] \\
& -2 X_{\gamma}\left[\frac{1}{g_{x y}^{2}}\left(h-u \frac{g_{z}}{g_{\text {spec }}}\right)\left(k-v \frac{g_{z}}{g_{\text {spec }}}\right)+\frac{u v}{g_{\text {spec }}^{2}}\right] .
\end{aligned}
$$

From three independent Bragg peak series, the parameters $Z_{a}^{2}$, $Z_{b}^{2}$ and $X_{\gamma}$ can be determined by solving the following set of equations:

$$
\left(\begin{array}{lll}
f_{11}\left(h_{1}\right) & f_{12}\left(k_{1}\right) & f_{13}\left(h_{1}, k_{1}\right) \\
f_{21}\left(h_{2}\right) & f_{22}\left(k_{2}\right) & f_{23}\left(h_{2}, k_{2}\right) \\
f_{31}\left(h_{3}\right) & f_{32}\left(k_{3}\right) & f_{33}\left(h_{3}, k_{3}\right)
\end{array}\right)\left(\begin{array}{c}
Z_{a}^{2} \\
Z_{b}^{2} \\
X_{\gamma}
\end{array}\right)=\left(\begin{array}{l}
1 \\
1 \\
1
\end{array}\right),
$$

where

$$
\begin{gathered}
f_{i 1}\left(h_{i}\right)=\frac{1}{g_{x y i}^{2}}\left(h_{i}-u \frac{g_{z i}}{g_{\text {spec }}}\right)^{2}+\frac{u^{2}}{g_{\text {spec }}^{2}} \\
f_{i 2}\left(k_{i}\right)=\frac{1}{g_{x y i}^{2}}\left(k_{i}-v \frac{g_{z i}}{g_{\text {spec }}}\right)^{2}+\frac{v^{2}}{g_{\text {spec }}^{2}}, \\
f_{i 3}\left(h_{i}, k_{i}\right)=-2\left[\frac{1}{g_{x y i}^{2}}\left(h_{i}-u \frac{g_{z i}}{g_{\text {spec }}}\right)\left(k_{i}-v \frac{g_{z i}}{g_{\text {spec }}}\right)+\frac{u v}{g_{\text {spec }}^{2}}\right]
\end{gathered}
$$

and $i=1,2$ and 3 . For obtaining $a, b$ and $\gamma$ from equations (39) to (41) the following identity is helpful:

$$
z_{a}^{2} z_{b}^{2} \frac{\sin ^{2} \gamma}{g_{\text {spec }}^{2}}=\frac{Z_{a}^{2} Z_{b}^{2}-X_{\gamma}^{2}}{g_{\text {spec }}^{2}-\left(u^{2} Z_{a}^{2}+v^{2} Z_{b}^{2}-2 u v X_{\gamma}\right)} .
$$




\section{APPENDIX C}

Compact expressions for the reciprocal cell parameters $a^{*}, b^{*}$ and $c^{*}$

If $h v-k u=0$ and $u \neq 0$ equations (22) and (24) can be reduced to

$$
\frac{g_{x y}^{2} g_{\text {spec }}^{2}}{c^{* 2}}=\left(u^{2} z_{a}^{2}+v^{2} z_{b}^{2}-2 u v z_{a} z_{b} \cos \gamma\right)\left(\frac{h}{u} w-l\right)^{2}
$$

and

$g_{x y}^{2} g_{\text {spec }}^{2}=\left(u^{2} z_{a}^{2}+v^{2} z_{b}^{2}-2 u v z_{a} z_{b} \cos \gamma\right)\left[g_{x y}^{2}+\left(\frac{h}{u} g_{\text {spec }}-g_{z}\right)^{2}\right]$,

respectively. Combining equations (48) and (49) the following expression can be obtained:

$$
\left(\frac{h}{u} g_{\text {spec }}-g_{z}\right)^{2}+g_{x y}^{2}=c^{* 2}\left(\frac{h}{u} w-l\right)^{2} .
$$

If $h v=k u$ and $v \neq 0$ the following relation can be deduced in an analogous way:

$$
\left(\frac{k}{v} g_{\text {spec }}-g_{z}\right)^{2}+g_{x y}^{2}=c^{* 2}\left(\frac{k}{v} w-l\right)^{2} .
$$

For $h w=l u$ and $k w=l v$ analogous formulas for $b^{*}$ and $a^{*}$, which contain only one real unknown besides the integer variables, are valid.

\section{APPENDIX $D$}

Useful expressions for determining the Laue indices

From equation (42) the following expression for the Laue index $k$ can be derived:

$$
\begin{aligned}
k= & v \frac{g_{z}}{g_{\text {spec }}}+\left(h-u \frac{g_{z}}{g_{\text {spec }}}\right) \frac{X_{\gamma}}{Z_{b}^{2}} \\
& \pm\left\{\left(1-\frac{u^{2} z_{a}^{2}+v^{2} z_{b}^{2}-2 u v z_{a} z_{b} \cos \gamma}{g_{\text {spec }}^{2}}\right)\right. \\
& \left.\times\left[\frac{g_{x y}^{2}}{Z_{b}^{2}}-\left(h-u \frac{g_{z}}{g_{\text {spec }}}\right)^{2} \frac{z_{a}^{2} z_{b}^{2} \sin ^{2} \gamma}{Z_{b}^{4}}\right]\right\}^{1 / 2} .
\end{aligned}
$$

The included components are given in equations (6), (40) and (41). As a term under a root sign must not be negative the following conditions need to be satisfied:

$$
\left|h-u \frac{g_{z}}{g_{\text {spec }}}\right| \leq g_{x y}\left[\left(\frac{a}{2 \pi}\right)^{2}-\frac{u^{2}}{g_{\text {spec }}^{2}}\right]^{1 / 2} \leq \frac{g_{x y}}{2 \pi} a .
$$

Note that analogous conditions are valid for $k$ and $l$ with the proper parameters $v, b$ and $w, c$, respectively. Furthermore, rough lower limits for the lattice parameters $a, b$ and $c$ with $u\left(2 \pi / g_{\text {spec }}\right) \leq a, v\left(2 \pi / g_{\text {spec }}\right) \leq b$ and $w\left(2 \pi / g_{\text {spec }}\right) \leq c$ result.

\section{APPENDIX $E$}

General rotation and analysis for three-component scattering vectors

In the general case, when the individual components of the reciprocal vector $g_{x}, g_{y}$ and $g_{z}$ have to be considered, equation (18) has to be expanded by including an additional rotation around the $[0,0,1]$ axis applying the matrix $\mathbf{R}(\varphi)$ :

$$
\mathbf{R}(\varphi)=\left(\begin{array}{ccc}
\cos \varphi & -\sin \varphi & 0 \\
\sin \varphi & \cos \varphi & 0 \\
0 & 0 & 1
\end{array}\right)
$$

which performs a rotation counterclockwise by an angle $\varphi$. Then the reciprocal vector

$$
\mathbf{g}=\left(\begin{array}{l}
g_{x} \\
g_{y} \\
g_{z}
\end{array}\right)
$$

can be expressed as

$$
\mathbf{g}=\mathbf{R}(\varphi) \mathbf{R}(\psi, \Phi) \mathbf{A}_{001}^{*}\left(\begin{array}{l}
h \\
k \\
l
\end{array}\right) .
$$

From equation (55), using equation (4), it follows that

$$
\left(\begin{array}{l}
h \\
k \\
l
\end{array}\right)=\mathbf{A}_{001}^{*}{ }^{-1} \mathbf{R}(\psi, \Phi)^{\mathrm{T}} \mathbf{R}(\varphi)^{\mathrm{T}} \mathbf{g}=\frac{1}{2 \pi} \mathbf{A}_{001} \mathbf{R}(\psi, \Phi)^{\mathrm{T}} \mathbf{R}(\varphi)^{\mathrm{T}} \mathbf{g} .
$$

With

$$
\mathbf{A}=\mathbf{A}_{001} \mathbf{R}(\psi, \Phi)^{\mathrm{T}} \mathbf{R}(\varphi)^{\mathrm{T}}
$$

equation (56) can be equivalently expressed as

$$
\mathbf{A g}=\left(\begin{array}{l}
\mathbf{a} \\
\mathbf{b} \\
\mathbf{c}
\end{array}\right) \mathbf{g}=2 \pi\left(\begin{array}{l}
h \\
k \\
l
\end{array}\right),
$$

where $\mathbf{a}, \mathbf{b}$ and $\mathbf{c}$ are the rotated unit-cell vectors with the relations $|\mathbf{a}|=a,|\mathbf{b}|=b,|\mathbf{c}|=c, \mathbf{a} \cdot \mathbf{b} /(|\mathbf{a}||\mathbf{b}|)=\cos \gamma, \mathbf{a} \cdot \mathbf{c} /(|\mathbf{a}||\mathbf{c}|)=$ $\cos \beta$ and $\mathbf{b} \cdot \mathbf{c} /(|\mathbf{b} \| \mathbf{c}|)=\cos \alpha$. These vectors can be written explicitly as

$$
\mathbf{a}=a\left(\begin{array}{c}
r_{a} \cos \left(\varphi+\psi-\Omega_{a}\right) \\
r_{a} \sin \left(\varphi+\psi-\Omega_{a}\right) \\
\sin \Phi \sin \psi
\end{array}\right)
$$

$$
\mathbf{b}=b\left(\begin{array}{c}
r_{b} \cos \left(\varphi+\psi+\Omega_{b}\right) \\
r_{b} \sin \left(\varphi+\psi+\Omega_{b}\right) \\
\sin \Phi \sin (\psi-\gamma)
\end{array}\right)
$$

$$
\mathbf{c}=c\left(\begin{array}{c}
r_{c} \cos \left(\varphi+\psi+\Omega_{c}\right) \\
r_{c} \sin \left(\varphi+\psi+\Omega_{c}\right) \\
\cos \Phi \sin \beta \sin \alpha^{*}+\sin \Phi\left(\cos \psi \sin \beta \cos \alpha^{*}+\sin \psi \cos \beta\right)
\end{array}\right),
$$

with

$$
r_{a}=\left(1-\sin ^{2} \Phi \sin ^{2} \psi\right)^{1 / 2},
$$




$$
\begin{gathered}
\cos \Omega_{a}=\frac{1}{r_{a}} \cos \psi, \\
r_{b}=\left[1-\sin ^{2} \Phi \sin ^{2}(\psi-\gamma)\right]^{1 / 2}, \\
\cos \Omega_{b}=\frac{1}{r_{b}} \cos (\psi-\gamma),
\end{gathered}
$$

$r_{c}=\left\{1-\left[\cos \Phi \sin \beta \sin \alpha^{*}+\sin \Phi\left(\cos \psi \sin \beta \cos \alpha^{*}\right.\right.\right.$

$$
\left.+\sin \psi \cos \beta)]^{2}\right\}^{1 / 2}
$$

$$
\cos \Omega_{c}=\frac{1}{r_{c}}\left(\cos \psi \cos \beta-\sin \psi \sin \beta \cos \alpha^{*}\right) .
$$

Thus, if three reciprocal vectors $\mathbf{g}_{1}, \mathbf{g}_{2}$ and $\mathbf{g}_{3}$ are given, the following relation is valid:

$$
\mathbf{G}\left(\begin{array}{l}
\mathbf{a} \\
\mathbf{b} \\
\mathbf{c}
\end{array}\right)^{\mathrm{T}}=\mathbf{G} \mathbf{A}^{\mathrm{T}}=2 \pi \mathbf{H}^{\mathrm{T}}
$$

where

$$
\mathbf{G}=\left(\begin{array}{lll}
g_{x 1} & g_{y 1} & g_{z 1} \\
g_{x 2} & g_{y 2} & g_{z 2} \\
g_{x 3} & g_{y 2} & g_{z 3}
\end{array}\right)
$$

and $\left(h_{i}, k_{i}, l_{i}\right)$ are the corresponding triples of Laue indices with

$$
\mathbf{H}=\left(\begin{array}{lll}
h_{1} & h_{2} & h_{3} \\
k_{1} & k_{2} & k_{3} \\
l_{1} & l_{2} & l_{3}
\end{array}\right)
$$

Equation (68) can be equivalently expressed as

$$
\mathbf{A}^{\mathrm{T}}=2 \pi \mathbf{G}^{-1} \mathbf{H}^{\mathrm{T}}
$$

Furthermore, as

$$
V=|\operatorname{det}(\mathbf{A})|=\operatorname{det}\left(\mathbf{A}_{001}\right)
$$

the following relation for the determinants of $\mathbf{G}$ and $\mathbf{H}$ is valid:

$$
|\operatorname{det}(\mathbf{G})| V=(2 \pi)^{3}|\operatorname{det}(\mathbf{H})| \text {. }
$$

If the specular scan $g_{\text {spec }}$ is known it is convenient to apply equations (15)-(17) in the rotation matrix $\mathbf{R}(\psi, \Phi)$ [equation (35)]. Then the unit-cell vectors $\mathbf{a}, \mathbf{b}$ and $\mathbf{c}$ can be expressed as given in Table 7 . Note that the $z$ components are only a function of the Miller index and $g_{\text {spec }}$.

The unit-cell vectors must be solutions to all reciprocal vectors $\mathbf{g}_{i}$, which, according to equation (58) and comprising equation (71), can be written as

$$
\mathbf{A g}_{i}=2 \pi\left[\mathbf{G}^{-1} \mathbf{H}^{\mathrm{T}}\right]^{\mathrm{T}} \mathbf{g}_{i}=2 \pi \mathbf{H}\left[\mathbf{G}^{-1}\right]^{\mathrm{T}} \mathbf{g}_{i}=2 \pi \mathbf{h}_{i},
$$

where

$$
\mathbf{g}_{i}=\left(\begin{array}{l}
g_{x i} \\
g_{y i} \\
g_{z i}
\end{array}\right)
$$

and

$$
\mathbf{h}_{i}=\left(\begin{array}{c}
h_{i} \\
k_{i} \\
l_{i}
\end{array}\right)
$$

Therefore, if $\mathbf{a}^{\prime}, \mathbf{b}^{\prime}$ and $\mathbf{c}^{\prime}$ are vectors of a superlattice with

$$
\mathbf{A}^{\prime}=\left(\begin{array}{l}
\mathbf{a}^{\prime} \\
\mathbf{b}^{\prime} \\
\mathbf{c}^{\prime}
\end{array}\right)=\mathbf{N A}
$$

where $\mathbf{N}$ is the transformation matrix [see equations (26)(29)], the following relation with $\mathbf{h}_{i}^{\prime}=\left(h_{i}^{\prime}, k_{i}^{\prime}, l_{i}^{\prime}\right)^{\mathrm{T}}$ can be derived from equation (74):

$$
\mathbf{A}^{\prime} \mathbf{g}_{i}=\mathbf{N A} \mathbf{g}_{i}=2 \pi \mathbf{N h} \mathbf{h}_{i}=2 \pi \mathbf{h}_{i}^{\prime}
$$

From equation (71) it can be deduced that $2 \pi \mathbf{G}^{-1} \mathbf{m}$, the product of the inverse matrix of three reciprocal vectors with a vector $\mathbf{m}$, consisting of a triple of arbitrary integers $\left(m_{1}, m_{2}\right.$, $\left.m_{3}\right)$, leads to a vector of the reduced cell, if $\mathbf{m}$ matches $\left(h_{1}, h_{2}\right.$, $\left.h_{3}\right)^{\mathrm{T}},\left(k_{1}, k_{2}, k_{3}\right)^{\mathrm{T}}$ or $\left(l_{1}, l_{2}, l_{3}\right)^{\mathrm{T}}$. If a transformation matrix $\mathbf{N}$ exists so that $\mathbf{m}$ equals $\mathbf{N}\left(h_{1}, h_{2}, h_{3}\right)^{\mathrm{T}}, \mathbf{N}\left(k_{1}, k_{2}, k_{3}\right)^{\mathrm{T}}$ or $\mathbf{N}\left(l_{1}, l_{2}\right.$, $\left.l_{3}\right)^{\mathrm{T}}$ a vector of a superlattice is obtained. According to equation (73) it is favourable to select three reciprocal vectors whose matrix results in a determinant, which is as small as possible but unequal to zero. Otherwise a unit cell which is too small and does not satisfy equation (74) for all reciprocal vectors may result. The Buerger and subsequently the reduced cell is obtained by choosing the three shortest vectors which are not coplanar and whose scalar products with all reciprocal vectors yield integers.

\section{Acknowledgements}

The experiments were performed at beamline W1, HASYLAB at DESY, Hamburg, Germany.

\section{Funding information}

Financial support was given by the Austrian Science Foundation FWF: [P30222].

\section{References}

Baskin, Y. \& Meyer, L. (1955). Phys. Rev. 100, 544.

Blöchl, P. E. (1994). Phys. Rev. B, 50, 17953-17979.

Bouchoms, I. P. M., Schoonveld, W. A., Vrijmoeth, J. \& Klapwijk, T. M. (1999). Synth. Met. 104, 175-178.

Boultif, A. \& Louër, D. (1991). J. Appl. Cryst. 24, 987-993.

Boultif, A. \& Louër, D. (2004). J. Appl. Cryst. 37, 724-731.

Breiby, D. W., Bunk, O., Andreasen, J. W., Lemke, H. T. \& Nielsen, M. M. (2008). J. Appl. Cryst. 41, 262-271.

Buerger, M. J. (1957). Z. Kristallogr. 109, 42-60.

David, W. I. F., Shankland, K., McCusker, L. B. \& Bärlocher, C. (2006). Structure Determination from Powder Diffraction Data. Oxford University Press.

Dejoie, C., Smeets, S., Baerlocher, C., Tamura, N., Pattison, P., Abela, R. \& McCusker, L. B. (2015). IUCrJ, 2, 361-370.

De Wolff, P. M. (2016). International Tables for Crystallography, 6th ed., Vol. A, pp. 709-713. Chichester: Wiley.

Djuric, T., Ules, T., Gusenleitner, S., Kayunkid, N., Plank, H., Hlawacek, G., Teichert, C., Brinkmann, M., Ramsey, M. \& Resel, R. (2012). Phys. Chem. Chem. Phys. 14, 262-272.

Dzyabchenko, A. V., Zavodnik, V. E. \& Belsky, V. K. (1979). Acta Cryst. B35, 2250-2253. 
Ehmann, H. M. A. \& Werzer, O. (2014). Cryst. Growth Des. 14, 36803684.

Giacovazzo, C. (2011). Editor. Fundamentals of Crystallography, 3rd ed. Oxford University Press.

Gildea, R. J., Waterman, D. G., Parkhurst, J. M., Axford, D., Sutton, G., Stuart, D. I., Sauter, N. K., Evans, G. \& Winter, G. (2014). Acta Cryst. D70, 2652-2666.

Hailey, A. K., Hiszpanski, A. M., Smilgies, D.-M. \& Loo, Y.-L. (2014). J. Appl. Cryst. 47, 2090-2099.

Heffelfinger, C. J. \& Burton, R. L. (1960). J. Polym. Sci. 47, 289-306.

Himes, V. L. \& Mighell, A. D. (1987). Acta Cryst. A43, 375-384.

Jacobson, R. A. (1976). J. Appl. Cryst. 9, 115-118.

Jiang, Z. (2015). J. Appl. Cryst. 48, 917-926.

Jones, A. O. F., Chattopadhyay, B., Geerts, Y. H. \& Resel, R. (2016). Adv. Funct. Mater. 26, 2233-2255.

Jones, A. O. F., Röthel, C., Lassnig, R., Bedoya-Martínez, O. N., Christian, P., Salzmann, I., Kunert, B., Winkler, A. \& Resel, R. (2017). CrystEngComm, 19, 1902-1911.

Krauss, T. N., Barrena, E., Zhang, X. N., de Oteyza, D. G., Major, J., Dehm, V., Würthner, F., Cavalcanti, L. P. \& Dosch, H. (2008). Langmuir, 24, 12742-12744.

Kresse, G. \& Furthmüller, J. (1996a). Comput. Mater. Sci. 6, 15-50.

Kresse, G. \& Furthmüller, J. (1996b). Phys. Rev. B, 54, 11169-11186.

Kresse, G. \& Hafner, J. (1993). Phys. Rev. B, 47, 558-561.

Kresse, G. \& Hafner, J. (1994). Phys. Rev. B, 49, 14251-14269.

Kresse, G. \& Joubert, D. (1999). Phys. Rev. B, 59, 1758-1775.

Křivý, I. \& Gruber, B. (1976). Acta Cryst. A32, 297-298.

Kroll, H., Stöckelmann, D. \& Heinemann, R. (2011). J. Appl. Cryst. 44, 812-819.

Mannsfeld, S. C. B., Tang, M. L. \& Bao, Z. (2011). Adv. Mater. 23, 127-131.

Mighell, A. D. (1976). J. Appl. Cryst. 9, 491-498.

Monkhorst, H. J. \& Pack, J. D. (1976). Phys. Rev. B, 13, 5188-5192.

Morawiec, A. (2017). J. Appl. Cryst. 50, 647-650.

Moser, A. (2012). PhD thesis, University of Technology, Graz, Austria.
Nam, H. J., Kim, Y. J. \& Jung, D. (2010). Bull. Korean Chem. Soc. 31, 2413-2415.

Niggli, P. (1928). Handbuch der Experimentalphysik, Vol. 7, Part 1. Leipzig: Akademische Verlagsgesellschaft.

Perdew, J. P., Burke, K. \& Ernzerhof, M. (1996). Phys. Rev. Lett. 77, 3865-3868.

Plimpton, S. (1995). J. Comput. Phys. 117, 1-19.

Powell, H. R. (1999). Acta Cryst. D55, 1690-1695.

Resel, R., Bainschab, M., Pichler, A., Dingemans, T., Simbrunner, C., Stangl, J. \& Salzmann, I. (2016). J. Synchrotron Rad. 23, 729-734.

Roe, R. J. \& Krigbaum, W. R. (1964). J. Chem. Phys. 40, 2608-2615.

Röthel, C. (2017). PhD thesis, University of Technology, Graz, Austria.

Salzmann, I. et al. (2012). ACS Nano, 6, 10874-10883.

Salzmann, I., Nabok, D., Oehzelt, M., Duhm, S., Moser, A., Heimel, G., Puschnig, P., Ambrosch-Draxl, C., Rabe, J. P. \& Koch, N. (2011). Cryst. Growth Des. 11, 600-606.

Salzmann, I. \& Resel, R. (2004). J. Appl. Cryst. 37, 1029-1033.

Santoro, A. \& Mighell, A. D. (1970). Acta Cryst. A26, 124-127.

Santoro, A., Mighell, A. D. \& Rodgers, J. R. (1980). Acta Cryst. A36, 796-800.

Schiefer, S., Huth, M., Dobrinevski, A. \& Nickel, B. (2007). J. Am. Chem. Soc. 129, 10316-10317.

Shmueli, U. (2006). Editor. International Tables for Crystallography, Vol. B, 2nd ed. Dordrecht: Springer.

Smilgies, D.-M. \& Blasini, D. R. (2007). J. Appl. Cryst. 40, 716-718.

Smith, G. S. \& Snyder, R. L. (1979). J. Appl. Cryst. 12, 60-65.

Tkatchenko, A., DiStasio, R. A., Car, R. \& Scheffler, M. (2012). Phys. Rev. Lett. 108, 236402.

Truger, M., Roscioni, O. M., Röthel, C., Kriegner, D., Simbrunner, C., Ahmed, R., Głowacki, E. D., Simbrunner, J., Salzmann, I., Coclite, A. M., Jones, A. O. \& Resel, R. (2016). Cryst. Growth Des. 16, $3647-3655$.

Vanommeslaeghe, K., Hatcher, E., Acharya, C., Kundu, S., Zhong, S., Shim, J., Darian, E., Guvench, O., Lopes, P., Vorobyov, I. \& Mackerell, A. D. (2010). J. Comput. Chem. 31, 671-690. 